\title{
Rational Recursion Operators for Integrable Differential-Difference Equations
}

\author{
Sylvain Carpentier ${ }^{1}$, Alexander V. Mikhailov ${ }^{3}$, Jing Ping Wang ${ }^{2}$ \\ 1 Mathematics Department, Columbia University, New York, USA \\ 2 School of Mathematics, Statistics and Actuarial Science, University of Kent, Canterbury, UK \\ 3 Applied Mathematics Department, University of Leeds, Leeds, UK. E-mail: A.V.Mikhailov@leeds.ac.uk
}

Received: 2 June 2018 / Accepted: 20 June 2019

Published online: 19 August 2019 - () The Author(s) 2019

\begin{abstract}
In this paper we introduce the concept of preHamiltonian pairs of difference operators, demonstrate their connections with Nijenhuis operators and give a criteria for the existence of weakly nonlocal inverse recursion operators for differential-difference equations. We begin with a rigorous setup of the problem in terms of the skew field of rational (pseudo-difference) operators over a difference field with a zero characteristic subfield of constants and the principal ideal ring of matrix rational (pseudo-difference) operators. In particular, we give a criteria for a rational operator to be weakly nonlocal. A difference operator is called preHamiltonian, if its image is a Lie subalgebra with respect to the Lie bracket on the difference field. Two preHamiltonian operators form a preHamiltonian pair if any linear combination of them is preHamiltonian. Then we show that a preHamiltonian pair naturally leads to a Nijenhuis operator, and a Nijenhuis operator can be represented in terms of a preHamiltonian pair. This provides a systematic method to check whether a rational operator is Nijenhuis. As an application, we construct a preHamiltonian pair and thus a Nijenhuis recursion operator for the differential-difference equation recently discovered by Adler and Postnikov. The Nijenhuis operator obtained is not weakly nonlocal. We prove that it generates an infinite hierarchy of local commuting symmetries. We also illustrate our theory on the well known examples including the Toda, the Ablowitz-Ladik, and the Kaup-Newell differential-difference equations.
\end{abstract}

\section{Introduction}

The existence of an infinite hierarchy of commuting symmetries is one of a characteristic property of integrable systems. Symmetries can be generated by recursion operators $[1,2]$, which are often pseudo-differential and map a symmetry to a new symmetry. An important property of recursion operators, called the Nijenhuis property, is to generate an abelian Lie algebra of symmetries. Such property has been independently studied by Fuchssteiner [3] and Magri [4]. To prove that a pseudo-differential operator is a 
Nijenhuis operator and it generates an infinite hierarchy of local symmetries is a challenging problem. In the most common case of weakly nonlocal Nijenhuis operators this problem has been addressed in [5-7]. The relations between bi-Hamiltonian structures and Nijenhuis operators have been studied in papers of Gel'fand and Dorfman [8,9] and Fuchssteiner and Fokas $[10,11]$. Recently a rigorous approach to pseudo-differential Hamiltonian operators have been developed in the series of papers by Barakat, De Sole, Kac and Valeri [12-14].

The theory of integrable differential-difference equations is much less developed. The basic concepts for symmetries, conservation laws and Hamiltonian operators were formulated in the frame of a variational complex in [15]. The aim of this paper is to build up a rigorous setting for rational matrix (pseudo-difference) operators suitable for the study of integrable differential-difference systems. We introduce and study preHamiltonian pairs of difference operators, their connections with Nijenhuis operators and the existence of weakly nonlocal inverse recursion operators for differential-difference equations.

Let us consider the well-known Volterra chain

$$
u_{t}=u\left(u_{1}-u_{-1}\right),
$$

where $u$ is a function of a lattice variable $n \in \mathbb{Z}$ and time $t$. Here we use the notations

$$
u_{t}=\partial_{t}(u), \quad u_{j}=\mathcal{S}^{j} u(n, t)=u(n+j, t)
$$

and $\mathcal{S}$ is the shift operator. It possesses a recursion operator

$$
R=u \mathcal{S}+u+u_{1}+u \mathcal{S}^{-1}+u\left(u_{1}-u_{-1}\right)(\mathcal{S}-1)^{-1} \frac{1}{u},
$$

where $(\mathcal{S}-1)^{-1}$ stands for the inverse of $\mathcal{S}-1$. Thus this operator is only defined on $u \operatorname{Im}(\mathcal{S}-1)$. It is a Nijenhuis operator and generates a commutative hierarchy of symmetries:

$$
u_{t_{j}}=R^{j}\left(u_{t}\right)=R^{j}\left(u\left(u_{1}-u_{-1}\right)\right), \quad j=0,1,2, \ldots
$$

The concept of Hamiltonian pairs was introduced by Magri [16]. He found that some systems admitted two distinct but compatible Hamiltonian structures (a Hamiltonian pair) and named them twofold Hamiltonian system, nowadays known as bi-Hamiltonian systems. The Volterra chain is a bi-Hamiltonian system and it can be written

$$
u_{t}=H_{1} \delta_{u} u=H_{2} \delta_{u} \frac{\ln u}{2},
$$

where $\delta_{u}$ is variational derivative with respect to the dependent variable $u$ and two difference operators

$$
\begin{aligned}
& H_{1}=u\left(\mathcal{S}-\mathcal{S}^{-1}\right) u ; \\
& H_{2}=u\left(\mathcal{S} u \mathcal{S}+u \mathcal{S}+\mathcal{S} u-u \mathcal{S}^{-1}-\mathcal{S}^{-1} u-\mathcal{S}^{-1} u \mathcal{S}^{-1}\right) u
\end{aligned}
$$

form a Hamiltonian pair. The Nijenhuis recursion operator of the Volterra chain can be obtained via the Hamiltonian pair, that is, $R=H_{2} H_{1}^{-1}$. This decomposition is known as the Lenard scheme used to construct the hierarchies of infinitely many symmetries and cosymmetries. 
Notice that the above difference operators have a right common factor:

$$
H_{1}=u(\mathcal{S}-1)\left(1+\mathcal{S}^{-1}\right) u ; \quad H_{2}=u(1+\mathcal{S})\left(u \mathcal{S}-\mathcal{S}^{-1} u\right)\left(1+\mathcal{S}^{-1}\right) u .
$$

This implies that

$$
R=A B^{-1} \text {, where } A=u(\mathcal{S}+1)\left(u \mathcal{S}-\mathcal{S}^{-1} u\right) \text { and } B=u(\mathcal{S}-1) .
$$

Here operators $A$ and $B$ are not skew-symmetric, and thus not Hamiltonian. However, like in the case of Hamiltonian pairs, the image of $A$ and $B$, as well as the image of linear combinations of these two operators, form a Lie subalgebra. Such operators we call preHamiltonian operator. In this paper, we explore properties of such operators and their relations with Nijenhuis operators. For the differential case some of these results have been obtained in [17]. The main difference between differential operators and difference operators lies in that the total derivative is a derivation and the shift operator $\mathcal{S}$ is an automorphism. The set of invertible difference operators is much richer than in the differential case. In the scalar case all difference operators of the form $a \mathcal{S}^{j}$, where $a$ is a difference function and $j \in \mathbb{Z}$, are invertible, while in the differential case, the only invertible operators are operators of multiplication by a function. The definition of the order of difference and differential operators are essentially different.

The arrangement of this paper is as follows: In Sect. 2, we define a difference field $\mathrm{F}$, the Lie algebra $\mathcal{A}$ of its evolutionary derivations (or evolutionary vector fields) which is a subalgebra of Der F and discuss algebraic properties of the noncommutative ring of difference operators. In particular, we show that it is a right and left Euclidean domain and satisfies the right (left) Ore property. Then we define the skew field of rational (pseudo-difference) operators, i.e. operators of the form $A B^{-1}$, where $A$ and $B$ are difference operators. Next we discuss the relation between rational operators and weakly nonlocal operators, namely we formulate a criteria for a rational operator to be weakly nonlocal. Finally we adapt all these results to rational matrix difference operators by defining the order of the operator as the order of its Dieudonné determinant. In Sect. 3 we define preHamiltonian difference operators as operators on $\mathrm{F}$ whose images define a Lie subalgebra in $\mathcal{A}$. We explore the interrelation between preHamiltonian pairs and Nijenhuis operators. We show that if operators $A$ and $B$ form a preHamiltonian pair, then $R=A B^{-1}$ is Nijenhuis. Conversely, if $R$ is Nijenhuis and $B$ is preHamiltonian, then $A$ and $B$ form a preHamiltonian pair. These two sections are the theoretical foundation of the paper. In Sect. 4, we give basic definitions such as symmetries, recursion operators and Hamiltonian for differential-difference equations. We also show how operators $A$ and $B$ are related to the equation if $A B^{-1}$ is its recursion operator. In the next two sections we apply the theoretical results in Sects. 2 and 3 to integrable differentialdifference equations. In Sect. 5, we construct a recursion operator for a new integrable equation derived by Adler and Postnikov in [18]:

$$
u_{t}=u^{2}\left(u_{2} u_{1}-u_{-1} u_{-2}\right)-u\left(u_{1}-u_{-1}\right),
$$

using its Lax representation presented in the same paper. The obtained recursion operator is no longer weakly nonlocal. We show that it is indeed Nijenhuis by rewriting it as a rational difference operator and that it generates infinitely many commuting local symmetries. To improve the readability, we put some technical lemmas used for the proof of the main result on the locality of commuting symmetries in "Appendix B". For some integrable differential-difference equations, such as the Ablowitz-Ladik Lattice [19], the recursion operator and its inverse are both weakly nonlocal. In Sect. 6, we apply 
the theoretical results from Sect. 2 to check whether the inverse recursion operators are weakly nonlocal, and if so, we demonstrate how to cast them in the weakly nonlocal form. To illustrate the method we choose four typical examples. However, the method is general and it can be applied to any integrable differential-difference system, including all systems listed in [20]. At the end of the paper we give a short conclusion and discussion on our new results on relation between preHamiltonian and Hamiltonian operators. To be self-contained, we also include "Appendix A", containing some basic definitions for a unital non-commutative ring.

\section{Algebraic Properties of Difference Operators}

In this section, we give a definition of rational difference operators and explore their properties. The main objects of our study in this paper are systems of evolutionary differential-difference equations and hidden structures associated with them. We first consider the scalar case. A generalization to the multi-component case will be discussed in the end of this section.

2.1. Difference field and its derivations. Let $\mathrm{k}$ be a zero characteristic base field, such as $\mathbb{C}$ or $\mathbb{R}$. We define the polynomial ring

$$
\mathrm{K}=\mathrm{k}\left[\ldots, u_{-1}, u_{0}, u_{1}, \ldots\right]
$$

of the infinite set of variables $\{u\}=\left\{u_{k} ; k \in \mathbb{Z}\right\}$ and the corresponding field of fractions

$$
\mathrm{F}=\mathrm{k}\left(\ldots, u_{-1}, u_{0}, u_{1}, \ldots\right) .
$$

It is assumed that every element of $\mathrm{K}$ and $\mathrm{F}$ depends on a finite number of variables only. We will denote $F^{\star}$ the subset of nonzero elements $F^{\star}=F \backslash\{0\}$ of $F$.

There is a natural automorphism $\mathcal{S}$ of the field $\mathrm{F}$, which we call the shift operator, defined as

$$
\mathcal{S}: a\left(u_{k}, \ldots, u_{r}\right) \mapsto a\left(u_{k+1}, \ldots, u_{r+1}\right), \quad \mathcal{S}: \alpha \mapsto \alpha, \quad a\left(u_{k}, \ldots, u_{r}\right) \in \mathrm{F}, \quad \alpha \in \mathrm{k} .
$$

For $a=a\left(u_{k}, \ldots, u_{r}\right) \in \mathrm{F}$ we will often use notation

$$
a_{i}=\mathcal{S}^{i}(a)=a\left(u_{k+i}, \ldots, u_{r+i}\right), \quad i \in \mathbb{Z},
$$

and omit index zero at $a_{0}$ or $u_{0}$ when there is no ambiguity. The field $\mathrm{F}$ equipped with the automorphism $\mathcal{S}$ is a difference field and the base field $\mathrm{k}$ is its subfield of constants.

The reflection $\mathcal{T}$ of the lattice $\mathbb{Z}$ defined by

$\mathcal{T}: a\left(u_{k}, \ldots, u_{r}\right) \mapsto a\left(u_{-k}, \ldots, u_{-r}\right), \quad \mathcal{T}: \alpha \mapsto \alpha, \quad a\left(u_{k}, \ldots, u_{r}\right) \in \mathrm{F}, \quad \alpha \in \mathrm{k}$,

is another obvious automorphism of $\mathrm{F}$ and $\mathrm{K}$. The composition $\mathcal{S} \mathcal{T} \mathcal{S}=\mathrm{Id}$ is the identity map. Thus the automorphisms $\mathcal{S}, \mathcal{T}$ generate the infinite dihedral group $\mathbb{D}_{\infty}$ and the subgroup generated by $\mathcal{S}$ is normal.

The automorphism $\mathcal{T}$ defines a $\mathbb{Z}_{2}$ grading of the difference field $\mathrm{F}$ (and $\operatorname{ring} \mathrm{K} \subset \mathrm{F}$ ):

$$
\mathrm{F}=\mathrm{F}_{0} \oplus \mathrm{F}_{1}, \quad \mathrm{~F}_{0} \cdot \mathrm{F}_{0}=\mathrm{F}_{0}, \quad \mathrm{~F}_{0} \cdot \mathrm{F}_{1}=\mathrm{F}_{1}, \quad \mathrm{~F}_{1} \cdot \mathrm{F}_{1}=\mathrm{F}_{0},
$$

where $\mathrm{F}_{k}=\left\{a \in \mathrm{F} \mid \mathcal{T}(a)=(-1)^{k} a\right\}$. 
Partial derivatives $\frac{\partial}{\partial u_{i}}, i \in \mathbb{Z}$ are commuting derivations of $F$ satisfying the conditions

$$
\mathcal{S} \frac{\partial}{\partial u_{i}}=\frac{\partial}{\partial u_{i+1}} \mathcal{S}, \quad \mathcal{T} \frac{\partial}{\partial u_{i}}=\frac{\partial}{\partial u_{-i}} \mathcal{T} .
$$

A derivation of $\mathrm{F}$ is said to be evolutionary if it commutes with the shift operator $\mathcal{S}$. Such derivation is completely determined by one element of $f \in \mathrm{F}$ and is of the form

$$
X_{f}=\sum_{i \in \mathbb{Z}} \mathcal{S}^{i}(f) \frac{\partial}{\partial u_{i}}, \quad f \in \mathrm{F} .
$$

An element $f$ is called the characteristic of the evolutionary derivation $X_{f}$. The action of $X_{f}(a)$ for $a \in \mathrm{F}$ can also be represented in the form

$$
X_{f}(a)=a_{*}[f],
$$

where $a_{*}[f]$ is the Fréchet derivative of $a=a\left(u_{p}, \ldots, u_{q}\right)$ in the direction $f$ defined as

$$
a_{*}[f]:=\left.\frac{d}{d \epsilon} a\left(u_{p}+\epsilon f_{p}, \ldots, u_{q}+\epsilon f_{q}\right)\right|_{\epsilon=0}=\sum_{i=p}^{q} \frac{\partial a}{\partial u_{i}} f_{i} .
$$

The Fréchet derivative of $a=a\left(u_{p}, \ldots, u_{q}\right)$ is a difference operator represented by a finite sum

$$
a_{*}=\sum_{i=p}^{q} \frac{\partial a}{\partial u_{i}} \mathcal{S}^{i}
$$

It is obvious that

$$
(\mathcal{T} a)_{*}=\sum_{i=p}^{q} \mathcal{T}\left(\frac{\partial a}{\partial u_{i}}\right) \mathcal{S}^{-i}
$$

Evolutionary derivations form a Lie subalgebra $\mathcal{A}$ in the the Lie algebra Der F. Indeed,

$$
\begin{aligned}
& \alpha X_{f}+\beta X_{g}=X_{\alpha f+\beta g}, \quad \alpha, \beta \in \mathrm{k}, \\
& {\left[X_{f}, X_{g}\right]=X_{[f, g]},}
\end{aligned}
$$

where $[f, g] \in \mathrm{F}$ denotes the Lie bracket

$$
[f, g]=X_{f}(g)-X_{g}(f)=g_{*}[f]-f_{*}[g] .
$$

Lie bracket (6) is k-bilinear, anti-symmetric and satisfies the Jacobi identity. Thus F, equipped with the bracket (6), has a structure of a Lie algebra over k.

The reflection $\mathcal{T}$ acts naturally on evolutionary vector derivations

$$
\mathcal{T}: X_{f} \mapsto X_{\mathcal{T}(f)}=\mathcal{T} \cdot X_{f} \cdot \mathcal{T}
$$

Thus the $\mathcal{A}$ is a graded Lie algebra

$$
\mathcal{A}=\mathcal{A}_{0} \oplus \mathcal{A}_{1}, \quad\left[\mathcal{A}_{0}, \mathcal{A}_{0}\right] \subset \mathcal{A}_{0},\left[\mathcal{A}_{0}, \mathcal{A}_{1}\right] \subset \mathcal{A}_{1},\left[\mathcal{A}_{1}, \mathcal{A}_{1}\right] \subset \mathcal{A}_{0},
$$

where $\mathcal{A}_{k}=\left\{X \in \mathcal{A} \mid \mathcal{T}(X)=(-1)^{k} X\right\}$. 
2.2. Rational difference operators. In this section we give definitions of difference operators and rational pseudo-difference operators, which for simplicity we shall call rational operators. We refer to the "Appendix A" for general results and definitions related to principal ideal domains. Although Corollary 1 and the first part of Proposition 2 follow directly from Proposition 1 in the abstract setting of Euclidean domains, we provide complete proofs for the sake of completeness.

Definition 1. A difference operator $B$ of order ord $B:=(M, N)$ with coefficients in $\mathrm{F}$ is a finite sum of the form

$$
\begin{aligned}
B= & b^{(N)} \mathcal{S}^{N}+b^{(N-1)} \mathcal{S}^{N-1}+\cdots+b^{(M)} \mathcal{S}^{M}, \quad b^{(N)} b^{(M)} \neq 0, b^{(k)} \in \mathrm{F}, \\
& M \leq N, N, M \in \mathbb{Z} .
\end{aligned}
$$

The total order of $B$ is defined as $\operatorname{Ord} B=N-M$. The total order of the zero operator is minus infinity Ord $0:=-\infty$ by definition.

The Fréchet derivative (5) is an example of a difference operator of order $(p, q)$ and total order Ord $a_{*}=q-p$. For an element $f \in \mathrm{F}$ the order and total order are defined as ord $f_{*}$ and Ord $f_{*}$ respectively.

Difference operators form a unital ring $\mathcal{R}=\mathrm{F}\left[\mathcal{S}, \mathcal{S}^{-1}\right]$ of Laurent polynomials in $\mathcal{S}$ with coefficients in $\mathrm{F}$, where multiplication is defined by

$$
a \mathcal{S}^{n} \cdot b \mathcal{S}^{m}=a \mathcal{S}^{n}(b) \mathcal{S}^{n+m}=a b_{n} \mathcal{S}^{n+m} .
$$

This multiplication is associative, but non-commutative. The definitions of some basic concepts for a unital associative ring are presented in the "Appendix A".

From the above definition it follows that if $A$ is a difference operator of order ord $A=$ $(p, q)$, then ord $\left(\mathcal{S}^{n} \cdot A \cdot \mathcal{S}^{m}\right)=(p+n+m, q+n+m)$ and $\operatorname{Ord}\left(\mathcal{S}^{n} \cdot A \cdot \mathcal{S}^{m}\right)=\operatorname{Ord} A=$ $q-p$. For any $A, B \in \mathcal{R}$ we have $\operatorname{Ord}(A B)=\operatorname{Ord} A+\operatorname{Ord} B$. Thus the total order is homomorphisms of the multiplicative monoid $\mathcal{R}$ to $\mathbb{Z}_{\geq 0} \cup\{\infty\}$.

The reflection $\mathcal{T}$ can be extended to an automorphism of $\mathcal{R}$ given by

$$
\mathcal{T} \cdot a \mathcal{S}^{m} \cdot \mathcal{T}=\mathcal{T}(a) \mathcal{S}^{-m}
$$

and defines a grading of $\mathcal{R}$ as follows:

$$
\mathcal{R}=\mathcal{R}_{0} \oplus \mathcal{R}_{1}, \quad \mathcal{R}_{k}=\left\{A \in \mathcal{R} \mid \mathcal{T} \cdot A \cdot \mathcal{T}=(-1)^{k} A\right\}
$$

It is obvious that $\operatorname{Ord}(\mathcal{T} \cdot A \cdot \mathcal{T})=\operatorname{Ord} A$.

A difference operator which has only one term $a \mathcal{S}^{n}, a \in \mathrm{F}^{*}, n \in \mathbb{Z}$ is called a monomial difference operator. The set of monomial difference operators are of the form $a \mathcal{S}^{n}, a \neq 0$. They have total order equal to zero and are invertible in $\mathcal{R}$. Monomial difference operators equipped with multiplication (8) form a nonabelian group

$$
\mathcal{R}^{\star}=\left\{a \mathcal{S}^{n} \mid a \in \mathrm{F}^{\star}, n \in \mathbb{Z}\right\} .
$$

We will use the notation $\operatorname{LT}(B)$ for a monomial difference operator representing the leading term of a difference operator which is the naturally ordered sum. For the operator $B$ in (7), we have $\operatorname{LT}(B)=b^{(N)} \mathcal{S}^{N}$.

Proposition 1. The ring $\mathcal{R}$ is a right and left Euclidean domain. 
Proof. Let us show that $\mathcal{R}$ is a right Euclidean, that is, for any $A, B \in \mathcal{R}$ there exist unique $Q, R \in \mathcal{R}$ such that $A=B \cdot Q+R$ and either $R=0$ or Ord $R<\operatorname{Ord} B$. First we prove the existence of $Q, R$. If $A=0$, then we can take $Q=R=0$. If $A \neq 0$ and $\operatorname{Ord} A<\operatorname{Ord} B$, we can take $Q=0, R=A$. For $\operatorname{Ord} A \geq \operatorname{Ord} B$ we proceed by induction on Ord $A=0(=\operatorname{Ord} B)$, then $A=a \mathcal{S}^{N}, B=b \overline{\mathcal{S}}^{M}$ for some $N, M \in \mathbb{Z}$ and they are invertible. Thus $A=B B^{-1} A$ and we can take $R=0, Q=B^{-1} A=$ $\mathcal{S}^{-M}(a / b) \mathcal{S}^{N-M}$. Finally, consider the case Ord $A=n \geq 1$, Ord $B=m, n \geq m$ and assume that the statement is true for all operators $A$ with total order less than $n$. Let the leading terms $\operatorname{LT}(A)=a \mathcal{S}^{N}$ and $\operatorname{LT}(B)=b \mathcal{S}^{M}$. The difference operator $\hat{A}=A-B \cdot\left(b \mathcal{S}^{M}\right)^{-1} \cdot a \mathcal{S}^{N}$ has Ord $\hat{A}<\operatorname{Ord} A=n$. Hence we can use the induction assumption and find $\hat{Q}, \hat{R}$, such that $\hat{A}=B \hat{Q}+\hat{R}$ and either $\hat{R}=0$ or $\operatorname{Ord} \hat{R}<\operatorname{Ord} B$. Thus

$$
A-B \cdot\left(b \mathcal{S}^{M}\right)^{-1} \cdot a \mathcal{S}^{N}=B \hat{Q}+\hat{R}
$$

that is,

$$
A=B\left(\left(b \mathcal{S}^{M}\right)^{-1} \cdot a \mathcal{S}^{N}+\hat{Q}\right)+\hat{R} .
$$

Therefore $Q=\left(b \mathcal{S}^{M}\right)^{-1} \cdot a \mathcal{S}^{N}+\hat{Q}$ and $R=\hat{R}$. As for the uniqueness, if one has $B Q+R=B \tilde{Q}+\tilde{R}$ with $\operatorname{Ord} R<\operatorname{Ord} B, \operatorname{Ord} \tilde{R}<\operatorname{Ord} B$, then $B(Q-\tilde{Q})=\tilde{R}-R$. If $Q \neq \tilde{Q}$ we arrive to a contradiction since $\operatorname{Ord}(B(Q-\tilde{Q}))>\operatorname{Ord}(\tilde{R}-R)$. Thus $Q=\tilde{Q}$ and $R=\tilde{R}$. The proof of the left Euclidean property is similar.

Corollary 1. Every right (left) ideal of the ring $\mathcal{R}$ is principal and generated by a unique element $A \in \mathcal{R}$ of minimal possible order with the leading term $\operatorname{LT}(A)=1$.

Proof. The zero ideal is obviously principal, it is generated by 0 . Let $J \subset \mathcal{R}$ be a right ideal and $\hat{A} \in J$ be an element of least possible total order. The element $A=$ $\hat{A} \cdot \operatorname{LT}(\hat{A})^{-1} \in J$, is of the same total order and with the leading term $\operatorname{LT}(A)=1$. Then for any other element $B \in J$ we have $B=A Q+R$ with either $R=0$ or Ord $R<$ Ord $A$. Since $B \in J$, we conclude that $R=0$, otherwise Ord $R<\operatorname{Ord} A$, which is in contradiction with the assumption that $A$ has the least possible order. Such element $A$ is obviously unique. If we assume the existence of $\tilde{A} \in J, \operatorname{Ord} \tilde{A}=\operatorname{Ord} A, \operatorname{LT}(\tilde{A})=1$, then $A-\tilde{A} \in J$ and $\operatorname{Ord}(A-\tilde{A})<\operatorname{Ord} A$. The latter is in contradiction with the assumption that $A$ has the least possible order. In a similar way we show that $\mathcal{R}$ is a left principal ideal ring.

Proposition 2. The ring $\mathcal{R}$ satisfies the right (left) Ore property, that is, for any $A, B \in \mathcal{R}$ their exist $A_{1}, B_{1}$, not both equal to zero, such that $A B_{1}=B A_{1}$, (resp. $\left.B_{1} A=A_{1} B\right)$. In other words, the right (left) ideal $A \mathcal{R} \cap B \mathcal{R}$ (resp. $\mathcal{R} A \cap \mathcal{R} B$ ) is nontrivial. Its generator $M$ has total order $\operatorname{Ord} A+\operatorname{Ord} B-\operatorname{Ord} D$, where $D$ is the greatest left (resp. right) common divisor of $A$ and $B$.

Proof. Let us assume that $\operatorname{Ord} A \geq \operatorname{Ord} B$ (otherwise we swap and rename $A, B$ ). If $B=0$, then $B_{1}=0$. If $B \neq 0$, we prove the claim by induction on Ord $B$. We assume that the statement is true for any $B$ with $\operatorname{Ord} B<k$ and we will show that it is also true for any $B, \operatorname{Ord} B=k$. Since $\mathcal{R}$ is right Euclidean, there exist $Q, R$ such that $A=B Q+R$ and either $R=0$ or $\operatorname{Ord} R<\operatorname{Ord} B$. If $R=0$ we take $A_{1}=Q, B_{1}=1$ and we are done. Since Ord $R<k$, there exist $\hat{B}, \hat{R}$ such that $B \hat{R}=R \hat{B}, \operatorname{Ord} \hat{R} \leq \operatorname{Ord} R$ and $\operatorname{Ord} \hat{B} \leq \operatorname{Ord} B$. Thus

$$
A \hat{B}=(B Q+R) \hat{B} \Leftrightarrow A \hat{B}=B(Q \hat{B}+\hat{R})
$$


and we can take $A_{1}=Q \hat{B}+\hat{R}, B_{1}=\hat{B}$. Finally, we can see that $\operatorname{Ord} A_{1} \leq \operatorname{Ord} A$ and $\operatorname{Ord} B_{1} \leq \operatorname{Ord} B$. The proof of the left Ore property is similar.

We proved that for any $A, B \in \mathcal{R}$ not both zero, the ideal $\mathcal{I}=A \mathcal{R} \cap B \mathcal{R}$ is not trivial. Since $\mathcal{R}$ is both a right and left principal ideal ring, $\mathcal{I}$ is generated by a difference operator $M, \mathcal{I}=M \mathcal{R}$. In particular, $M=A B_{1}=B A_{1}$ for some difference operators $B_{1}$ and $A_{1}$. From the first part of the proof, we know that $\operatorname{Ord} M \leq \operatorname{Ord} A+\operatorname{Ord} B$. Let us assume that $A$ and $B$ are left coprime and that $\operatorname{Ord} M<\operatorname{Ord} A+\operatorname{Ord} B$. The ideal $\mathcal{J}=\mathcal{R} A_{1} \cap \mathcal{R} B_{1}$ is also nontrivial and generated by a difference operator $N$. We know that $\operatorname{Ord} N$ is at most $\operatorname{Ord} A_{1}+\operatorname{Ord} B_{1} . M$ is an element of $\mathcal{J}$ and $\operatorname{Ord} M=$ $\operatorname{Ord} A+\operatorname{Ord} B_{1}>\operatorname{Ord} A_{1}+\operatorname{Ord} B_{1} \geq \operatorname{Ord} N$, hence there exists a difference operator $C$ such that $M=C N$ and $\operatorname{Ord} C>0$. Let $A_{2}$ and $B_{2}$ be such that $A_{2} B_{1}=B_{2} A_{1}=N$. Then $A=C A_{2}$ and $B=C B_{2}$, which contradicts the hypothesis that $A$ and $B$ are left coprime.

The fact that $\mathcal{R}$ is a principal ideal domain gives sense to the notions of greatest common divisors and least common multiples (see "Appendix A"). The following lemma, which will be used in Proposition 13, relates the images of two difference operators to the image of their right least common multiple.

Lemma 1. Let $A$ and $B$ be two nonzero left coprime difference operators with coefficients in $\mathrm{F}$. Suppose that $A(x)=B(y)$ for some $x, y \in \mathrm{F}$. Let $M=A C=B D$ be their right least common multiple. Then, there exists $z \in \mathrm{F}$ such that $x=C(z)$ and $y=D(z)$. In particular $\operatorname{Im} A \cap \operatorname{Im} B=\operatorname{Im} M$.

Proof. By definition of $M, C$ and $D$ are right coprime. It follows from the Bezout's Lemma that there exist two difference operators $U$ and $V$ such that

$$
U C+V D=1
$$

Multiplying (9) on $D$ and on $C$ from the left we obtain

$$
\begin{aligned}
& D U C=(1-D V) D, \\
& C V D=(1-C U) C .
\end{aligned}
$$

By assumption $A$ and $B$ are left coprime therefore it follows from Lemma 5 (ii) that there exist two difference operators $P$ and $Q$ such that

$$
\begin{aligned}
& 1-D V=P B, \quad D U=P A \\
& 1-C U=Q A, \quad C V=Q B .
\end{aligned}
$$

Using the assumption $A(x)=B(y)$ and the first line of (12) we get

$$
y=(P B+D V)(y)=P A(x)+D V(y)=D(U(x)+V(y)),
$$

and similarly using the second line of (12) we get

$$
x=(C U+Q A)(x)=C U(x)+Q B(x)=C(U(x)+V(y)) .
$$

Hence, the statement holds with $z=U(x)+V(y)$.

The domain $\mathcal{R}$ can be naturally embedded in the skew field of rational pseudodifference operators, which we will call simply rational operators. 
Definition 2. A rational (pseudo-difference) operator $L$ is defined as $L=A B^{-1}$ for some $A, B \in \mathcal{R}$ and $B \neq 0$. The set of all rational operators is

$$
\mathcal{Q}=\left\{A B^{-1} \mid A, B \in \mathcal{R}, B \neq 0\right\} .
$$

Remark 1. The skew field $\mathcal{Q}$ is a minimal subfield of the skew field $\mathcal{Q}^{L}$ of the Laurent formal series

$$
\mathcal{Q}^{L}=\left\{\sum_{n=k}^{\infty} a^{(-n)} \mathcal{S}^{-n} \mid a^{(l)} \in \mathrm{F}, l \in \mathbb{Z}\right\}
$$

containing $\mathcal{R}$. As well as it is a minimal subfield of the skew field $\mathcal{Q}^{T}$ of the Taylor formal series

$$
\mathcal{Q}^{T}=\left\{\sum_{n=k}^{\infty} a^{(n)} \mathcal{S}^{n} \mid a^{(l)} \in \mathrm{F}, l \in \mathbb{Z}\right\}
$$

containing $\mathcal{R}$. The skewfields $\mathcal{Q}^{L}$ and $\mathcal{Q}^{T}$ are isomorphic. The isomorphism is given by the reflection map $\mathcal{T}$.

Proposition 3. Any rational operator $L=A B^{-1}$ can also be written in the form $L=$ $\hat{B}^{-1} \hat{A}$ with $\hat{A}, \hat{B} \in \mathcal{R}$ and $\hat{B} \neq 0$.

Proof. It follows from the Ore property that for any $A, B \in \mathcal{R}, B \neq 0$ there exist $\hat{A}, \hat{B} \in \mathcal{R}$ and $\hat{B} \neq 0$ such that $\hat{B} A=\hat{A} B$. Multiplying this expression on $\hat{B}$ from the left and $B^{-1}$ from the right we obtain $L=A B^{-1}=\hat{B}^{-1} \hat{A}$.

Thus any statement for the representation $L=A B^{-1}$ can be easily reformulated to the representation $L=\hat{B}^{-1} \hat{A}$. In particular,

$$
\mathcal{Q}=\left\{A B^{-1} \mid A, B \in \mathcal{R}, B \neq 0\right\}=\left\{B^{-1} A \mid A, B \in \mathcal{R}, B \neq 0\right\} .
$$

Proposition 4. $\mathcal{Q}$ is the skew field of rational operators over $\mathrm{F}$.

Proof. We need to show that the set $\mathcal{Q}$ is closed under addition and multiplication. Let $A, B, C, D \in \mathcal{R}$ with $B \neq 0, D \neq 0$. It follows from the Ore property that there exist nonzero $\hat{B}, \hat{D} \in \mathcal{R}$ such that $B \hat{D}=D \hat{B}$. Hence

$$
A B^{-1}+C D^{-1}=(A \hat{D}+C \hat{B}) \cdot(B \hat{D})^{-1} \in \mathcal{Q} .
$$

Also there exist nonzero $\hat{B}, \hat{C}$ such that $B \hat{C}=C \hat{B}$. Hence

$$
\left(A B^{-1}\right) \cdot\left(C D^{-1}\right)=(A \hat{C}) \cdot(D \hat{B})^{-1} \in \mathcal{Q}
$$

implying that $\mathcal{Q}$ is also closed under multiplication.

Proposition 5. The decomposition $L=A B^{-1}, A, B \in \mathcal{R}$ of an element $L \in \mathcal{Q}$ is unique if we require that $B$ has a minimal possible total order with leading term $\mathrm{LT}(B)=1$. For any other decomposition $L=\hat{A} \hat{B}^{-1}, \hat{A}, \hat{B} \in \mathcal{R}$ there exists $C \in \mathcal{R}$ such that $\hat{A}=A C, \hat{B}=B C$. Moreover, if $D^{-1} E$ is a (left) minimal decomposition of $L$, then $\operatorname{Ord} D=\operatorname{Ord} B$. 
Proof. For a given $L \in \mathcal{Q}$ the set

$$
J=\{X \in \mathcal{R} \mid L X \in \mathcal{R}\}
$$

is a right ideal in $\mathcal{R}$. Indeed, if $X, Y \in J$, then $L(X+Y)=L X+L Y \in \mathcal{R}$ meaning that $X+Y \in J$, and $J$ is stable under right multiplication by any element of $\mathcal{R}$. The ideal $J$ is principal, and according to Corollary 1 it is generated by a unique element $B$ of the least possible order, if we require that the leading term $\operatorname{LT}(B)=1$. Any other $\hat{B} \in J$ can be represented as $\hat{B}=B C$ where $C \in \mathcal{R}$, since $B$ is a generator of the principal right ideal $J$. By Proposition 2, we know that a generator $M$ of the left ideal generated by $A$ and $B$ has total order $\operatorname{Ord} A+\operatorname{Ord} B$. By definition of $M$ there exist left coprime difference operators $D$ and $E$ such that $D A=E B=M$. Therefore $D^{-1} E$ is a left minimal decomposition of $L$ and $\operatorname{Ord} D=\operatorname{Ord} B$.

The definition of total order for difference operators (Definition 1) can be extended to rational operators:

$\operatorname{Ord}\left(A B^{-1}\right):=\operatorname{Ord} A-\operatorname{Ord} B, \quad \operatorname{Ord}\left(\hat{B}^{-1} \hat{A}\right):=\operatorname{Ord} \hat{A}-\operatorname{Ord} \hat{B}, \quad A, B, \hat{A}, \hat{B} \in \mathcal{R}$.

Definition 3. A formal adjoint operator $A^{\dagger}$ for any $A \in \mathcal{Q}$ can be defined recursively:

1. $a^{\dagger}=a$ for any $a \in \mathrm{F}$,

2. $\mathcal{S}^{\dagger}=\mathcal{S}^{-1}$,

3. $(A+B)^{\dagger}=B^{\dagger}+A^{\dagger}$ for any $A, B \in \mathcal{Q}$,

4. $(A \cdot B)^{\dagger}=B^{\dagger} \cdot A^{\dagger}$ for any $A, B \in \mathcal{Q}$,

5. $\left(A^{-1}\right)^{\dagger}=\left(A^{\dagger}\right)^{-1}$ for any $A \in \mathcal{Q}$.

In particular, We say an operator $H \in \mathcal{Q}$ is skew-symmetric if $H^{\dagger}=-H$.

For example, we have

$$
\left(\left(\mathcal{S}+a \mathcal{S}^{-1}\right) \cdot(b-\mathcal{S})^{-1}\right)^{\dagger}=\left(b-\mathcal{S}^{-1}\right)^{-1} \cdot\left(\mathcal{S}^{-1}+\mathcal{S}(a) \mathcal{S}\right), \quad a, b \in \mathrm{F} .
$$

For any $A \in \mathcal{Q}$, if ord $A=(p, q)$ then ord $A^{\dagger}=(-q,-p)$. Obviously Ord $A^{\dagger}=\operatorname{Ord} A$.

2.3. Rational and weakly nonlocal difference operators. In the theory of integrable systems, the majority of $1+1$-dimensional integrable equations possesses weakly nonlocal [21] Nijenhuis recursion operators. For integrable differential-difference equations, weakly nonlocal operators are often rational operators with only a finite number of nonlocal terms of the form $a(\mathcal{S}-1)^{-1} b$, where $a, b \in \mathrm{F}$. In this section, we show how to write a weakly nonlocal operator as a rational operator and provide a way to test whether a rational operator is indeed weakly nonlocal. For the differential case, the answers are given by Lemma 4.5 in [17].

First we give a definition of the full kernel difference operators. We then prove that for such operators, their inverse are weakly nonlocal.

For a difference operator $A \in \mathcal{R}$ it is obvious that

$$
\operatorname{dim}_{\mathrm{k}} \operatorname{Ker} A \leq \operatorname{Ord} A \text {. }
$$

Indeed, if there is an element $a \in \mathrm{F}$ such that $a \in \operatorname{Ker} A$, then we can represent $A=\tilde{A}(\mathcal{S}-1) \frac{1}{a}$, where $\operatorname{Ord} \tilde{A}=\operatorname{Ord} A-1$. Zero total order difference operator is invertible and thus it has a trivial kernel space. A difference operator of a nonzero order may also have a trivial kernel in $\mathrm{F}$ as well. For example $\operatorname{Ker}_{\mathrm{k}}(\mathcal{S}-u)=0$ since equation $\mathcal{S}(v)=u v$ does not have a solution $v \in \mathrm{F}$. 
Definition 4. We say that a difference operator has a full kernel in F (is a full kernel operator) if the dimension of its kernel over the field $\mathrm{k}$ equals to the total order of the operator.

In what follows, we show how to construct a full kernel operator given the generators of its kernel and prove an important property of such operators.

Proposition 6. Assume that $f^{(1)}, \ldots, f^{(n)}$ are linearly independent over $\mathrm{k}$ in $\mathrm{F}$. Then there exists a full kernel difference operator $P \in \mathcal{R}$ such that the $f^{(i)}, i=1, \ldots, n$ span ker $P$.

Proof. We prove the statement by induction on $n$. If $n=1$, we define

$$
P=(\mathcal{S}-1) \frac{1}{f^{(1)}} .
$$

It is clear that $\operatorname{Ord} P=1$ and its kernel is spanned by $f^{(1)}$. Assume that $Q$ is a full kernel operator with Ord $Q=n-1$ and its kernel is spanned by $f^{(1)}, \ldots, f^{(n-1)}$. Since $f^{(i)}, i=1, \ldots, n$ are linearly independent, we have $Q\left(f^{(n)}\right) \neq 0$ by construction of $Q$. We define

$$
P=(\mathcal{S}-1) \frac{1}{Q\left(f^{(n)}\right)} Q .
$$

Clearly it is the required full kernel operator and its kernel is spanned by $f^{(1)}, \ldots$, $f^{(n)}$.

Remark 2. A difference operator $Q \in \mathcal{R}$ with full kernel spanned by the k-linearly independent elements $f^{(i)} \in \mathrm{F}, i=1, \ldots, n$, can be obtained using the determinant expression

$$
Q(g)=\operatorname{det}\left(\begin{array}{cccc}
f^{(1)} & \cdots & f^{(n)} & g \\
\mathcal{S}\left(f^{(1)}\right) & \cdots & \mathcal{S}\left(f^{(n)}\right) & \mathcal{S}(g) \\
\vdots & \vdots & \vdots & \vdots \\
\mathcal{S}^{n}\left(f^{(1)}\right) & \cdots & \mathcal{S}^{n}\left(f^{(n)}\right) & \mathcal{S}^{n}(g)
\end{array}\right) \text { for any } g \in \mathrm{F} .
$$

Proposition 7. The inverse operators of full kernel operators are weakly nonlocal.

Proof. We prove the statement by induced on the total order of such operator $B$. If $B$ is a full kernel operator with $\operatorname{Ord} B=1$, it can be written as $B=a \mathcal{S}^{i}(\mathcal{S}-1) b$ for some $i \in \mathbb{Z}$. Thus

$$
B^{-1}=\frac{1}{b} \mathcal{S}^{-i}(\mathcal{S}-1)^{-1} \frac{1}{a}
$$

is weakly nonlocal.

Let $B$ be a full kernel operator with the total order of $n$ and $a \in \operatorname{ker} B$. It follows from Proposition 6 that there is a full kernel operator $C$ with total order of $n-1$ such that

$$
B=C(\mathcal{S}-1) \frac{1}{a} .
$$

By the induction assumption, $C^{-1}$ is weakly nonlocal, that is, there exist two sets of linearly independent functions $b^{(i)}$ and $c^{(i)}, i=1, \ldots, n-1$ such that 


$$
C^{-1}=E+\sum_{i=1}^{n-1} b^{(i)}(\mathcal{S}-1)^{-1} c^{(i)}, \quad E \in \mathcal{R} .
$$

Multiplying $C$ on its left, we get

$$
\sum_{i=1}^{n-1} C\left(b^{(i)}\right)(\mathcal{S}-1)^{-1} c^{(i)}=0
$$

implying $b^{(i)} \in \operatorname{ker} C$. Note that for any $b^{(i)} \in \operatorname{ker} C, i=1, \ldots, n-1$, there exists $d^{(i)}$, which is in $\operatorname{ker} B$ such that $b^{(i)}=(\mathcal{S}-1) \frac{d^{(i)}}{a}$. Therefore, we have

$$
B^{-1}=a(\mathcal{S}-1)^{-1} C^{-1}=a(\mathcal{S}-1)^{-1}\left(E+\sum_{i=1}^{n-1}\left((\mathcal{S}-1) \frac{d^{(i)}}{a}\right)(\mathcal{S}-1)^{-1} c^{(i)}\right),
$$

whose nonlocal terms are

$$
a(\mathcal{S}-1)^{-1} E^{\dagger}(1)+\sum_{i=1}^{n-1}\left(d^{(i)}(\mathcal{S}-1)^{-1} c^{(i)}-a(\mathcal{S}-1)^{-1} \frac{c^{(i)} d_{1}^{(i)}}{a_{1}}\right),
$$

where we used the identity

$$
(\mathcal{S}-1)^{-1}\left(d_{1}-d\right)(\mathcal{S}-1)^{-1}=d(\mathcal{S}-1)^{-1}-(\mathcal{S}-1)^{-1} d_{1}, \quad d \in \mathrm{F} .
$$

This leads to the conclusion that $B^{-1}$ is weakly nonlocal.

We are now ready to prove the statement on the relation between the rational and weakly nonlocal difference operators.

Theorem 1. Let $R$ be a rational operator with minimal right fractional decomposition $A B^{-1}$ and $\operatorname{Ord} B=n$. Then the following three statements are equivalent:

(i) The operator $B$ has a full kernel in $\mathrm{F}$;

(ii) The operator $R$ is weakly nonlocal, that is, $R=L+\sum_{i=1}^{n} p^{(i)}(\mathcal{S}-1)^{-1} q^{(i)}$, where $L \in \mathcal{R}$, and $\left\{p^{(i)}, i=1, \ldots, n\right\}$ and $\left\{q^{(i)}, i=1, \ldots, n\right\}$ are two linearly independent sets over $\mathrm{k}$ in $\mathrm{F}$;

(iii) The operator $B^{\dagger}$ has a full kernel in $\mathrm{F}$.

Proof. The statement (i) $\Rightarrow$ (ii) directly follows from Proposition 7 since the multiplication of a difference operator and a weakly nonlocal operator is weakly nonlocal.

We now prove that (ii) $\Rightarrow$ (iii). Knowing

$$
R=A B^{-1}=L+\sum_{i=1}^{n} p^{(i)}(\mathcal{S}-1)^{-1} q^{(i)},
$$

we multiply it on the right by $B$ and obtain its nonlocal terms

$$
\sum_{i=1}^{n} p^{(i)}(\mathcal{S}-1)^{-1} B^{\dagger}\left(q^{(i)}\right)=0,
$$

which implies that all $q^{(i)}$, s are in the kernel of $B^{\dagger}$ and thus $n \leq \operatorname{dim}\left(\operatorname{ker} B^{\dagger}\right)$. 
Let $C$ be a common multiple of the difference operators $\frac{1}{q^{(i)}}(\mathcal{S}-1)$, that is, a difference operator such that for all $i$ there exists a difference operator $M^{(i)}$ satisfying $C=\frac{1}{q^{(i)}}(\mathcal{S}-$ 1) $M^{(i)}$. Thus we have

$$
R=L+\sum_{i=1}^{n} p^{(i)}(\mathcal{S}-1)^{-1} q^{(i)}=\left(L C+\sum_{i=1}^{n} p^{(i)} M^{(i)}\right) C^{-1}
$$

Since $A B^{-1}$ is a minimal right fractional decomposition for $R$, there exists a difference operator $D$ such that

$$
L C+\sum_{i=1}^{n} p^{(i)} M^{(i)}=A D \text { and } C=B D .
$$

This leads to $\operatorname{Ord} C=n \geq \operatorname{Ord} B$. Note that $\operatorname{Ord} B=\operatorname{Ord} B^{\dagger}$ and $\operatorname{Ord} B^{\dagger} \geq \operatorname{dim}\left(\operatorname{ker} B^{\dagger}\right)$. Therefore, we have

$$
\operatorname{Ord} B^{\dagger}=\operatorname{dim}\left(\operatorname{ker} B^{\dagger}\right)=n
$$

implying that $B^{\dagger}$ has a full kernel spanned by all $q^{(i)}$ 's.

Finally we prove that (iii) $\Rightarrow$ (i). It follows from Proposition 7 that the inverse of $B^{\dagger}$ is weakly nonlocal. Using the proof of (ii) $\Rightarrow$ (iii), we obtain that statement of (i).

From the proof of Theorem 1, we are able to specify the nonlocal terms for weakly nonlocal operator.

Corollary 2. Under the condition of Theorem 1 , for $R=L+\sum_{i=1}^{n} p^{(i)}(\mathcal{S}-1)^{-1} q^{(i)}$, the linearly independent functions $p^{(i)}$ 's span $A(\operatorname{ker} B)$ and the linearly independent functions $q^{(i)}$ 's span $\operatorname{ker} B^{\dagger}, i=1, \ldots, n$.

Following from this theorem, we are immediately able to get the statement for the inverse of rational operator:

Corollary 3. Let $R=A B^{-1}$ with $A, B \in \mathcal{R}$. Then $R^{-1}$ is weakly nonlocal if and only if $A$ has a full kernel in $\mathrm{F}$.

Corollary 2 combined with Proposition 6 provides us with a method to write a weakly nonlocal operator in the form of a rational operator $R=A B^{-1}$ : We first construct a full kernel operator $B^{\dagger}$ using $q^{(i)}$ 's. Then we have $A=R B$. We use such construction for the examples in Sect. 6, where we will also apply Corollary 3 to the recursion operators of integrable differential-difference equations to see whether their inverse operators are weakly nonlocal or not. If so, we are going to compute the seeds for symmetry and co-symmetry hierarchies (its nonlocal terms), that is, the $p^{(i)}$ 's and $q^{(i)}$ 's for $R^{-1}$ in the above theorem.

2.4. Matrix difference and rational pseudo-difference operators. We recall here some facts from linear algebra over non-commutative rings and skew fields, which is a specialisation of the general theory $[22,23]$ to the case of difference algebra (the ring $\mathcal{R}$ and skew field $\mathcal{Q}$ ). We denote by $\mathcal{M}_{n}(\mathcal{R})$ and $\mathcal{M}_{n}(\mathcal{Q})$ the rings of $n \times n$ matrices over the ring $\mathcal{R}$ and skew field $\mathcal{Q}$ respectively. Since $\mathcal{R}$ is a principal ideal ring, then the ring 
$\mathcal{M}_{n}(\mathcal{R})$ is also a principal ideal ring (see proof in [24], as well as the short and useful review of non-commutative principal ideal rings [25]).

Let $\mathcal{A}_{i}$ denote the $i$-th row of the matrix $\mathcal{A}$ and $\mathcal{A}_{i, j}$ denote the $(i, j)$ entry of $\mathcal{A}$. For $1 \leq i \neq j \leq n$ and arbitrary $B \in \mathcal{R}$ (or $B \in \mathcal{Q}$ ) the $\mathcal{R}$-elementary (resp. $\mathcal{Q}$-elementary) row operation $\tau_{i, j}(B)$ changes the row $\mathcal{A}_{i} \mapsto \mathcal{A}_{i}+B \cdot \mathcal{A}_{j}$ and leaves the other rows unchanged. The transformation $\tau_{i, j}(B)$ is invertible $\left(\tau_{i, j}(B) \tau_{i, j}(-B)=\mathrm{Id}\right)$ and can be represented by a multiplication from the left by the matrix $\tau_{i, j}(B)=I+B E_{i, j}$, where $I$ is the unit matrix and $E_{i, j}$ is the matrix with the $(i, j)$ entry equal to 1 and zero elsewhere. Note that the transformation $\sigma_{i, j}=\tau_{i, j}(1) \tau_{j, i}(-1) \tau_{i, j}(1)$ replaces $\mathcal{A}_{i}$ by $\mathcal{A}_{j}$ and $\mathcal{A}_{j}$ by $-\mathcal{A}_{i}$, leaving other rows unchanged.

$\mathcal{R}$-elementary row operations generate a group $\mathcal{E}_{n}(\mathcal{R})$, which is a subgroup of the group $G L_{n}(\mathcal{R})$ of invertible matrix difference operators. Similarly, $\mathcal{Q}$-elementary row operations generate a group $\mathcal{E}_{n}(\mathcal{Q})$, a subgroup of the group $G L_{n}(\mathcal{Q})$ of invertible matrix pseudo-difference operators.

Lemma 2. Let $\mathcal{A} \in \mathcal{M}_{n}(\mathcal{R})$. Then there exist two invertible matrices $\mathcal{U}$ and $\mathcal{V}$ such that $\mathcal{U} \mathcal{A} \mathcal{V}$ is diagonal.

Proof. Let $\mathcal{N}$ be an element of the set $E=\left\{\mathcal{U} \mathcal{A} \mathcal{V} \mid \mathcal{U}, \mathcal{V} \in \mathcal{E}_{n}(\mathcal{R})\right\}$ such that for all $\mathcal{M} \in E$, either $\mathcal{M}_{11}=0$ or Ord $\mathcal{N}_{11} \leq \operatorname{Ord} \mathcal{M}_{11}$. We claim that all entries in the first column of $\mathcal{N}$ are divisible on the right by $\mathcal{N}_{11}$. Otherwise, using elementary row operations which amounts to multiply $\mathcal{N}$ on the left by an invertible matrix, one can find $\mathcal{M} \in E$ such that $\mathcal{M}_{11} \neq 0$ and Ord $\mathcal{M}_{11}<\operatorname{Ord} \mathcal{N}_{11}$, which contradicts the definition of $\mathcal{N}$. Similarly, $\mathcal{N}_{11}$ must divide all the entries of the first row of $\mathcal{N}$ on the left. Therefore, there exist invertible matrix difference operators $\mathcal{U}$ and $\mathcal{V}$ such that $\mathcal{U N} \mathcal{V}$ has only zero entries in its first row and first column, apart from the first coefficient which is $\mathcal{N}_{11}$. We conclude by induction on $n$.

Proposition 8. Let $\mathcal{A} \in \mathcal{M}_{n}(\mathcal{R})$. Then it can be brought to a upper triangular form $\mathcal{A}^{\Delta}$ with $\mathcal{A}_{i, j}^{\Delta}=0$ for $i>j$ by $\mathcal{R}$-elementary row operations and

$$
\mathcal{A}^{\Delta}=\mathcal{G} \mathcal{A}, \quad \mathcal{G} \in \mathcal{E}_{n}(\mathcal{R})
$$

Proof. We prove the claim by induction on $n$. If $n=1$, the matrix is already in the form required. Now we assume that any matrix from $\mathcal{M}_{n-1}(\mathcal{R})$ can be brought to a upper triangular form by $\mathcal{R}$-elementary row transformation. Therefore the first $n-1$ rows of matrix $\mathcal{A}$ can be brought to the upper triangular form.

(i) If $\mathcal{A}_{n, 1}=0$, then by deleting the first row and the first column of $\mathcal{A}$ we reduce the problem to the case $\mathcal{M}_{n-1}(\mathcal{R})$ and we are done due to the induction hypothesis.

(ii) If $\mathcal{A}_{1,1}=0$, we use the transformation $\sigma_{1, n}$ to reduce the problem to the case (i).

(iii) The remaining case are $\mathcal{A}_{1,1} \neq 0, \mathcal{A}_{n, 1} \neq 0$. Suppose $\operatorname{Ord} \mathcal{A}_{n, 1} \leq \operatorname{Ord} \mathcal{A}_{1,1}$ (otherwise, we can swap the rows by the transformation $\sigma_{1, n}$ ). Then there exist $B, R \in \mathcal{R}$ such that $\mathcal{A}_{1,1}=B \cdot \mathcal{A}_{n, 1}+R$ and either $R=0$ or $\operatorname{Ord} R<\operatorname{Ord} \mathcal{A}_{n, 1}$ and we apply the transformation $\tau_{1, n}(-B)$ replacing $\mathcal{A}_{1}$ by $\hat{\mathcal{A}}_{1}=\mathcal{A}_{1}-B \mathcal{A}_{n}$. If $R=0$, then the updated row $\hat{\mathcal{A}}_{1}$ has zero entry $\hat{\mathcal{A}}_{1,1}=0$ and we are done (ii), or Ord $\hat{\mathcal{A}}_{1,1}<\operatorname{Ord} \mathcal{A}_{n, 1}$ and we use $\sigma_{1, n}$ to swap the rows. Iterating this procedure we can make the entry $(n, 1)$ vanish, reducing the problem to the first case (i).

The ring $\mathcal{M}_{n}(\mathcal{R}), n \geq 2$ has zero divisors. We will denote by $\mathcal{M}_{n}^{\times}(\mathcal{R})$ the multiplicative monoid of regular elements, i.e. the elements which are not zero divisors. A 
difference matrix operator $\mathcal{A}$ is regular if and only if its upper triangular form $\mathcal{A}^{\Delta}$ is regular, i.e. if and only if

$$
\delta \in \tau\left(\mathcal{A}^{\Delta}\right):=\mathcal{A}_{1,1}^{\Delta} \cdot \mathcal{A}_{2,2}^{\Delta} \cdots \mathcal{A}_{n, n}^{\Delta} \neq 0 .
$$

Definition 5. The total order of a matrix difference operator $\mathcal{A} \in \mathcal{M}_{n}(\mathcal{R})$ is defined as the sum of total orders of the diagonal entries of a corresponding upper triangular operator $\mathcal{A}^{\Delta}$, i.e.

$$
\operatorname{Ord} \mathcal{A}=\sum_{i=1}^{n} \operatorname{Ord} \mathcal{A}_{i, i}^{\triangle}=\operatorname{Ord} \delta \in \tau(\mathcal{A})
$$

Proposition 9. A difference matrix operator $\mathcal{A}$ is invertible in $\mathcal{M}_{n}(\mathcal{R})$ (i.e. $\mathcal{A}^{-1} \in$ $\mathcal{M}_{n}(\mathcal{R})$ and thus $\mathcal{A} \in G L_{n}(\mathcal{R})$ ), if and only if $\operatorname{Ord} \mathcal{A}=0$.

Proof. If Ord $\mathcal{A}=0$, then all entries on the diagonal part $\mathcal{A}_{d}^{\Delta}:=\operatorname{diag}\left(\left(\mathcal{A}_{1,1}^{\Delta}\right), \ldots\right.$, $\left(\mathcal{A}_{n, n}^{\Delta}\right)$ ) of $\mathcal{A}^{\Delta}$ have total order zero and thus invertible. Multiplying $\mathcal{A}^{\Delta}$ on the left by matrix $\left(\mathcal{A}_{d}^{\triangle}\right)^{-1}$ we obtain an upper triangular matrix $\tilde{\mathcal{A}}^{\Delta}=\left(\mathcal{A}_{d}^{\triangle}\right)^{-1} \mathcal{G} \mathcal{A}$ with the unit matrix on the diagonal. By induction on $n$ it is easy to show that there is a composition of $\mathcal{R}$-elementary row transformations $\tilde{\mathcal{G}}$ such that $\tilde{\mathcal{G}} \tilde{\mathcal{A}}^{\Delta}=I$. If $n=1$ there is nothing to do. We assume the existence of the inverse matrix in $\mathcal{M}_{n-1}(\mathcal{R})$. The entries $\tilde{\mathcal{A}}_{k, n}^{\Delta}, k=1, \ldots, n-1$ of the last column can be set to zero by the transformation $\prod_{k=1}^{n-1} \tau_{k, n}\left(-\tilde{\mathcal{A}}_{k, n}^{\Delta}\right) \cdot \tilde{\mathcal{A}}^{\Delta}$ which reduces the problem to the case in $\mathcal{M}_{n-1}(\mathcal{R})$. The necessity is obvious from the consideration of a diagonal matrix $\mathcal{A}$.

Example 1. Let us consider the following matrix difference operator

$$
\mathcal{A}=\left(\begin{array}{cc}
1 & \mathcal{S}^{2} \\
a_{-1} \mathcal{S}^{-1} & a_{1} \mathcal{S}
\end{array}\right)
$$

where $a \in \mathrm{F}, a_{k}=\mathcal{S}^{k}(a)$ and $a_{i} \neq a_{j}$ if $i \neq j$. The transformation $\mathcal{A} \mapsto \mathcal{A}^{\triangle}=$ $\tau_{2,1}\left(-a_{-1} \mathcal{S}^{-1}\right) \mathcal{A}$ brings $\mathcal{A}$ to an upper triangular form and $\delta \in \tau\left(\mathcal{A}^{\triangle}\right)=\left(a_{1}-a_{-1}\right) \mathcal{S}$. Thus Ord $\mathcal{A}=0$ and the inverse matrix difference operator of $\mathcal{A}$ exists. Indeed,

$$
\mathcal{A}^{-1}=\left(\begin{array}{cc}
\frac{a_{2}}{a_{2}-a} & -\frac{1}{a_{2}-a} \mathcal{S} \\
-\frac{a_{-2}}{a-a_{-2}} \mathcal{S}^{-2} & \frac{1}{a-a_{-2}} \mathcal{S}^{-1}
\end{array}\right) .
$$

If we use a different sequence of elementary row transformations

$$
\mathcal{A} \mapsto \tilde{\mathcal{A}}^{\Delta}=\tau_{1,2}\left(-\frac{1}{a_{2}} \mathcal{S}\right) \tau_{2,1}\left(-\frac{a_{1} a_{-1}}{a_{1}-a_{-1}} \mathcal{S}^{-1}\right) \mathcal{A},
$$

which also brings the difference matrix operator $\mathcal{A}$ to an upper triangular form, then $\delta \in \tau\left(\tilde{\mathcal{A}}^{\Delta}\right)=\frac{a_{1}\left(a_{2}-a\right)}{a_{2}} \mathcal{S}$, but the total order of $\mathcal{A}$ does not depend on the choice of the sequence $\operatorname{Ord} A=\operatorname{Ord} \delta \in \tau\left(\tilde{\mathcal{A}}^{\triangle}\right)=0$ (see below). 
The correctness of Definition 5, i.e. the independence of $\operatorname{Ord} \mathcal{A}$ from the choice of row transformations, can be justified by the theory of Dieudonné determinants $\left(\Delta_{n}\right)$ (in the case of skew polynomial rings it has been discussed in [26]). The above definition of total order for matrix difference operators is a restriction of the map Ord $\Delta_{n}: \mathcal{M}_{n}(\mathcal{Q}) \mapsto$ $\mathbb{Z} \cup\{\infty\}$ to $\operatorname{Ord} \Delta_{n}: \mathcal{M}_{n}(\mathcal{R}) \mapsto \mathbb{Z}_{\geq 0} \cup\{\infty\}$. This observation results in a simpler way to compute the total order of matrix difference operators by treating them as elements of $\mathcal{M}_{n}(\mathcal{Q})$.

The Dieudonné determinant $\Delta_{n}$ is defined for matrices with entries in an arbitrary skew field $\mathbb{K}$ (see $[22,23,27])$. In our case the skew field is $\mathbb{K}=\mathcal{Q}$ and we are dealing with matrix rational operators $\mathcal{M}_{n}(\mathcal{Q})$, but what is presented below is equally applicable to rational operators or any skew field of fraction of a left principal ideal domain. The Dieudonné determinant is a map from $\mathcal{M}_{n}(\mathcal{Q})$ to $\overline{\mathcal{Q}}=\mathcal{Q}^{\times} / \mathcal{Q}^{(1)}$ or zero, where $\mathcal{Q}^{\times}$ is the multiplicative group of nonzero elements of $\mathcal{Q}$, and $\mathcal{Q}^{(1)}$ denotes the commutator subgroup $\mathcal{Q}^{(1)}=\left[\mathcal{Q}^{\times}, \mathcal{Q}^{\times}\right] \subset \mathcal{Q}^{\times}$, which is normal. The group $\mathcal{Q}^{(1)}$ is generated by elements of the form $A B A^{-1} B^{-1}, A, B \in \mathcal{Q}^{\times}$. The quotient group $\overline{\mathcal{Q}}$ is commutative and its elements are cosets $A \mathcal{Q}^{(1)}, A \in \mathcal{Q}^{\times}$. There is a natural projection $\pi: \mathcal{Q}^{\times} \mapsto \overline{\mathcal{Q}}$ given by $\pi(A)=\bar{A}:=A \mathcal{Q}^{(1)}$ for any $A \in \mathcal{Q}^{\times}$.

Dieudonné has shown that $\mathcal{E}_{n}(\mathcal{Q})$ is a normal subgroup of $G L_{n}(\mathcal{Q})$ and that there is a group isomorphism $\Delta_{n}: G L_{n}(\mathcal{Q}) / \mathcal{E}_{n}(\mathcal{Q}) \mapsto \overline{\mathcal{Q}}$ given by a map $\Delta_{n}$ (Theorem 1 . in [27]), which is now called the Dieudonné determinant. The function $\Delta_{n}: \mathcal{M}_{n}(\mathcal{Q}) \mapsto \overline{\mathcal{Q}}$ is:

1. multiplicative: $\Delta_{n}(\mathcal{A B})=\Delta_{n}(\mathcal{A}) \Delta_{n}(\mathcal{B})$;

2. if $\mathcal{A} \in \mathcal{E}_{n}(\mathcal{Q})$, then $\Delta_{n}(\mathcal{A})=\overline{1}$;

3. if $\mathcal{A}^{\prime}$ is obtained from $\mathcal{A}$ by multiplying one row of $\mathcal{A}$ on the left by $B \in \mathcal{Q}$, then

$$
\Delta_{n} \mathcal{A}^{\prime}=\bar{B} \cdot \Delta_{n} \mathcal{A}
$$

4. if a matrix $\mathcal{A}$ is degenerate (i.e. one row is a left $\mathcal{Q}$-linear combination of other rows), then $\Delta_{n}(\mathcal{A})=0$.

In order to find $\Delta_{n} \mathcal{A}$ for $\mathcal{A} \in \mathcal{M}_{n}(\mathcal{Q})$ one can use the algorithm given by Dieudonné [27] (see also $§ 1, \mathrm{Ch}$. IV [22]), or use the Bruhat normal form approach ( $\$ 20$, Part III, [23]). A simple way to find the Dieudonné determinant of a matrix $\mathcal{A} \in \mathcal{M}_{n}(\mathcal{Q})$ is to use a composition of $\mathcal{Q}$-elementary row transformations in order to bring the matrix $\mathcal{A}$ to a upper triangular form $\mathcal{A}^{\triangle}=\mathcal{G} \mathcal{A}, \mathcal{G} \in \mathcal{E}_{n}(\mathcal{Q})$, then multiply the diagonal entries of $\mathcal{A}^{\triangle}$ (in an arbitrary order) and apply the projection $\pi$ to the result

$$
\Delta_{n}(\mathcal{A})=\pi\left(\prod_{k=1}^{n} \mathcal{A}_{k, k}^{\Delta}\right) .
$$

It follows from [27] that $\Delta_{n}(\mathcal{A})$ does not depend on the choice of elementary row transformations, neither on the order in the product of diagonal elements of $\mathcal{A}^{\Delta}$.

It follows from Definition 1 and (15) that $\operatorname{Ord} P=0$ for any $P \in \mathcal{Q}^{(1)}$, thus function Ord has a constant value on a coset and the map

$$
\text { Ord : } \overline{\mathcal{Q}} \mapsto \mathbb{Z}
$$

is defined correctly.

Definition 6. The total order of a matrix rational operator $\mathcal{A} \in \mathcal{M}_{n}(\mathcal{Q})$ is

$$
\operatorname{Ord} \mathcal{A}:=\operatorname{Ord} \Delta_{n}(\mathcal{A}) \text {. }
$$


In the case of difference operators $\mathcal{A} \in \mathcal{M}_{n}(\mathcal{R})$ we have defined a function $\delta \in \tau\left(\mathcal{A}^{\Delta}\right) \in$ $\mathcal{R}$ (17). Although the value of this function depends on the choice of $\mathcal{R}$-elementary row transformations, its natural projection to $\overline{\mathcal{Q}}$ does not, since it coincides with the Dieudonné determinant

$$
\pi\left(\delta \in \tau\left(\mathcal{A}^{\Delta}\right)\right)=\Delta_{n}(\mathcal{A}) .
$$

This restriction of the total order definition to the ring of matrix difference operators together with Proposition 9 results in the exact sequence of monoid homomorphisms (similar to Theorem 1.1 in [26]):

$$
1 \longmapsto G L_{n}(\mathcal{R}) \longmapsto \mathcal{M}_{n}(\mathcal{R}) \longmapsto \mathbb{Z}_{\geq 0} \cup\{\infty\} \longmapsto 0
$$

Definition 5 is a way to define the total order of a matrix difference operator, bypassing the skew field of rational operators, its quotient group $\overline{\mathcal{Q}}$ and the theory of Dieudonné determinants.

Note that the Dieudonné determinant and the total order of a matrix (rational) difference operator and the transposed matrix operator may not coincide. In the above Example (18):

$$
\Delta_{2}(\mathcal{A})=\pi\left(\left(a_{1}-a_{-1}\right) \mathcal{S}\right), \quad \Delta_{2}\left(\mathcal{A}^{t r}\right)=0 .
$$

A formally conjugated matrix (rational) difference operator has a usual definition, i.e. the corresponding matrix is transposed and each entry is formally conjugated: $\left(\mathcal{A}^{\dagger}\right)_{i, j}=$ $\left(\mathcal{A}_{j, i}\right)^{\dagger}$. For formally conjugated operators we have $\Delta_{n}\left(\mathcal{A}^{\dagger}\right)=\left(\Delta_{n} \mathcal{A}\right)^{\dagger}$ and therefore $\operatorname{Ord} \mathcal{A}^{\dagger}=\operatorname{Ord} \mathcal{A}$.

There are many ways to represent a matrix rational operator as a ratio of matrix difference operators. For example any $\mathcal{L} \in \mathcal{M}_{n}(\mathcal{Q})$ can be represented as

$$
\mathcal{L}=\hat{\mathcal{A}} \cdot \mathcal{D}^{-1}=\tilde{\mathcal{A}} \cdot M^{-1}, \quad \mathcal{D}=\operatorname{diag}\left(M_{1}, \ldots, M_{n}\right), M_{k}, M \in \mathcal{R} \backslash\{0\} .
$$

Indeed, the entries $\mathcal{L}_{i, j} \in \mathcal{Q}$ and thus $\mathcal{L}_{i, j}=A_{i, j} B_{i, j}^{-1}, A_{i, j}, B_{i, j} \in \mathcal{R}$. Since the ring $\mathcal{R}$ satisfies the Ore property (Proposition 2) there exists a least right common multiple $M_{i}$ of the elements $B_{1, i}, \ldots B_{n, i}$ and therefore there exist $P_{1, i}, \ldots P_{n, i} \in \mathcal{R}$ such that $M_{i}=$ $B_{1, i} P_{1, i}=\cdots=B_{n, i} P_{n, i}$. Taking $\hat{\mathcal{A}}_{i, j}=\mathcal{L}_{i, j} P_{i, j}$ we obtain the first representation. Let $M$ be the least right common multiple of $M_{1}, \ldots, M_{n}$. There exist $Q_{1}, \ldots Q_{n} \in \mathcal{R}$ such that $M=M_{1} Q_{1}=\cdots=M_{n} Q_{n}$, therefore $\tilde{A}=\hat{A} \cdot \operatorname{diag}\left(Q_{1}, \ldots, Q_{n}\right)$.

Since the ring of difference operators $\mathcal{R}$ is a principal ideal domain, the ring of matrices $\mathcal{M}_{n}(\mathcal{R})$ satisfies the left and right Ore property (see proof in [24]) and thus

$$
\begin{aligned}
\mathcal{M}_{n}(\mathcal{Q}) & =\left\{\mathcal{A B}^{-1} \mid(\mathcal{A}, \mathcal{B}) \in \mathcal{M}_{n}(\mathcal{R}) \times \mathcal{M}_{n}^{\times}(\mathcal{R})\right\} \\
& =\left\{\mathcal{B}^{-1} \mathcal{A} \mid(\mathcal{A}, \mathcal{B}) \in \mathcal{M}_{n}(\mathcal{R}) \times \mathcal{M}_{n}^{\times}(\mathcal{R})\right\} .
\end{aligned}
$$

A representation of matrix rational operators as right (left) fractions is not unique. However, once we clear the common right (resp. left) divisors, we get a minimal fraction, in the following sense:

Theorem 2. For any $\mathcal{L} \in \mathcal{M}_{n}(\mathcal{Q})$ there is a minimal right (resp. left) decomposition $\mathcal{L}=\mathcal{A B}^{-1}$ (resp. $\mathcal{L}=\hat{\mathcal{B}}^{-1} \hat{\mathcal{A}}$ ) with $\mathcal{A}, \mathcal{B}$ right (resp. $\hat{\mathcal{A}}, \hat{\mathcal{B}}$ left) coprime. Any other right decomposition $\mathcal{L}=\mathcal{A}_{1} \mathcal{B}_{1}^{-1}$ (resp. left decomposition $\mathcal{L}=\hat{\mathcal{A}}_{1}^{-1} \hat{\mathcal{B}}_{1}$ ) is of the from $\mathcal{A}_{1}=\mathcal{A} \cdot \mathcal{C}, \mathcal{B}_{1}=\mathcal{B} \cdot \mathcal{C}\left(\right.$ resp. $\left.\hat{\mathcal{A}}_{1}=\mathcal{C} \cdot \hat{\mathcal{A}}, \mathcal{B}_{1}=\mathcal{C} \cdot \hat{\mathcal{B}}\right)$, where $\mathcal{C} \in \mathcal{M}_{n}^{\times}(\mathcal{R})$. Moreover $\operatorname{Ord} \mathcal{B}=\operatorname{Ord} \hat{\mathcal{B}}$ and is minimal possible among all decompositions. 
Proof. We will first prove by induction on $n$ that if $A$ and $B$ are matrix difference operators of size $n \times n$ with $B$ regular, if $M$ is a generator of the right ideal $A \mathcal{M}_{n}(\mathcal{R}) \cap$ $B \mathcal{M}_{n}(\mathcal{R})$ and $N$ a greatest left common divisor of $A$ and $B$, then $\operatorname{Ord} A+\operatorname{Ord} B=$ $\operatorname{Ord} M+\operatorname{Ord} N$.

It is true for $n=1$ by Proposition 2. Let us now consider $A$ and $B$ of size $n+1$. Using invertible matrices we can assume that $A$ and $B$ are both upper triangular. Indeed, one can factorize them as $A=T_{A} U_{A}$ and $B=T_{B} U_{B}$ with $T_{A}, T_{B}$ upper triangular and $U_{A}, U_{B}$ invertible. Hence if there exist $C$ and $D$ such that $T_{A} D=T_{B} C$ with $\operatorname{Ord} D \leq \operatorname{Ord} T_{B}=\operatorname{Ord} B$, then we can write $A\left(U_{A}^{-1} D\right)=B\left(U_{B}^{-1} C\right)$. Let us consider $A$ and $B$ in block matrix form:

$$
A=\left(\begin{array}{ll}
E & X \\
0 & P
\end{array}\right), \quad B=\left(\begin{array}{ll}
F & Y \\
0 & Q
\end{array}\right),
$$

where $E$ and $F$ are of size $n \times n, P$ and $Q$ are difference operators and $X$ and $Y$ have size $n \times 1$. First, let $E G=F H$ be a generator of the right ideal $E \mathcal{M}_{n}(\mathcal{R}) \cap F \mathcal{M}_{n}(\mathcal{R})$ in $\mathcal{M}_{n}(\mathcal{R}), P \hat{Q}=Q \hat{P}$ be a generator of the right ideal $P \mathcal{R} \cap Q \mathcal{R}$ in $\mathcal{R}$ and $K$ be a generator of the right ideal $E \mathcal{M}_{n}(\mathcal{R})+F \mathcal{M}_{n}(\mathcal{R})$ in $\mathcal{M}_{n}(\mathcal{R})$ (which is also called the greatest left common divisor of $E$ and $F$ ). We have by the induction hypothesis $\operatorname{Ord} K=\operatorname{Ord} F-\operatorname{Ord} G$. One can find a difference operator $R$ with $\operatorname{Ord} R \leq \operatorname{Ord} K$ and a vector difference operator $Z$ such that $K Z=(Y \hat{P}-X \hat{Q}) R$. Indeed, by Lemma 2 one can assume that $K$ is a diagonal matrix $\operatorname{diag}\left(K_{0}, \ldots, K_{n}\right)$. Let us call by $L_{0}, \ldots, L_{n}$ the entries of the vector $Y \hat{P}-X \hat{Q}$. Then we can find for all $i=0, \ldots n$ difference operators $M_{i}$ and $N_{i}$ such that $\operatorname{Ord} N_{i} \leq \operatorname{Ord} K_{i}$ and $K_{i} M_{i}=L_{i} N_{i}$. Let $R$ be a generator of the right ideal $N_{0} \mathcal{R} \cap \cdots \cap N_{n} \mathcal{R}$. Then $\operatorname{Ord} R \leq \sum_{i=0}^{n} \operatorname{Ord} N_{i} \leq \sum_{i=0}^{n} \operatorname{Ord} K_{i}=\operatorname{Ord} K$ and there exists a vector $Z$ such that $K Z=L R$. Finally, by definition of $K$ there exist two matrix difference operator $V$ and $W$ such that $E V-F W=K$. Let

$$
C=\left(\begin{array}{cc}
H & W Z \\
0 & \hat{P} R
\end{array}\right), \quad D=\left(\begin{array}{cc}
G & V Z \\
0 & \hat{Q} R
\end{array}\right) .
$$

Then $\operatorname{Ord} D \leq \operatorname{Ord} B$ and $A D=B C$.

The proof of the remaining parts of the statement are identical to the scalar case, see the proofs of Propositions 2 and 5.

The inequality (16) is also true for a regular matrix difference operator $\mathcal{A} \in \mathcal{M}_{n}^{\times}(\mathcal{R})$ and we say that $\mathcal{A}$ is a full kernel operator if $\operatorname{Dim}_{\mathrm{k}} \operatorname{Ker} \mathcal{A}=\operatorname{Ord} \mathcal{A}$. Theorem 1 , Corollary 2 and Corollary 3 from the previous section are also true for matrix rational operators.

\section{PreHamiltonian Pairs and Nijenhuis Operators}

Zhiber and Sokolov, in their study of Liouville integrable hyperbolic equations [28], have discovered a family of special differential operators with the property that they define a new Lie bracket and are homomorphisms from the Lie algebra with the newly induced bracket to the original Lie algebra. These operators can be viewed as a generalization of Hamiltonian operators, although they are not necessarily skew-symmetric. Inspired by the work of Zhiber and Sokolov, infinite sequences of such scalar differential operators of arbitrary order were constructed in [29] using symbolic representation [30,31]. Kiselev and van de Leur gave some examples of such matrix differential operators [32] and investigated the geometric meaning of such operators. They named them preHamiltonian operators in [33] and defined the compatibility of two such operators. Recently, 
Carpentier renamed them as integrable pairs and investigated the interrelations between such pairs and Nijenhuis operators [17]. In principle, many results for differential operators also work for difference operators since $\mathcal{R}$ is a principal ideal domain. In this section, we develop further the theory of preHamiltonian operators and extend it to the difference case. Similarly to the previous section, we illustrate our results for the scalar case.

Definition 7. A difference operator $A$ is called preHamiltonian if $\operatorname{Im} A$ is a Lie subalgebra of $(\mathrm{F},[\bullet, \bullet])$, i.e. if

$$
[\operatorname{Im} A, \operatorname{Im} A] \subseteq \operatorname{Im} A .
$$

By a direct computation, it is easy to see ([29]) that an operator $A$ is preHamiltonian if and only if there exists a 2 -form on $\mathrm{F}$ denoted by $\omega_{A}$ such that

$$
A_{*}[A a](b)-A_{*}[A b](a)=A \omega_{A}(a, b) \text { for all } a, b \in \mathrm{F} .
$$

For a given $a \in \mathrm{F}$, both $\omega_{A}(a, \bullet)$ and $\omega_{A}(\bullet, a)$ are in $\mathcal{R}$, i.e. difference operators on $\mathrm{F}$.

For a Hamiltonian operator $H$, the Jacobi identity is equivalent to (cf. [9])

$$
[H a, H b]=H\left(b_{*}[H a]+(H a)_{*}^{\dagger}(b)-a_{*}[H b]+a_{*}^{\dagger}(H b)\right),
$$

for all $a, b \in \mathrm{F}$, where $\dagger$ is the adjoint of the operator. Clearly, Hamiltonian operators are preHamiltonian with $\omega_{H}(a, b)=(H a)_{*}^{\dagger}(b)+a_{*}^{\dagger}(H b)$. We are going to explore the relation between preHamiltonian pairs and Hamiltonian pairs in the forthcoming paper [34]. Here we look at their relations with Nijenhuis operators.

Similarly to Hamiltonian operators, in general, the linear combination of two preHamiltonian operators is no longer preHamiltonian. This naturally leads to the following definition:

Definition 8. We say that two difference operators $A$ and $B$ form a preHamiltonian pair if $A+\lambda B$ is preHamiltonian for all constant $\lambda \in \mathrm{k}$.

A preHamiltonian pair $A$ and $B$ implies the existence of 2-forms $\omega_{A}, \omega_{B}$ and $\omega_{A+\lambda B}=$ $\omega_{A}+\lambda \omega_{B}$. They satisfy

$$
\begin{gathered}
A_{*}[B a](b)+B_{*}[A a](b)-A_{*}[B b](a)-B_{*}[A b](a) \\
=A \omega_{B}(a, b)+B \omega_{A}(a, b) \text { for all } a, b \in \mathrm{F}
\end{gathered}
$$

Gel'fand and Dorfman [8] and Fuchssteiner and Fokas [10,11] discovered the relations between Hamiltonian pairs and Nijenhuis operators. These pairs naturally generate Nijenhuis operators. In what follows, we show that preHamiltonian pairs also give rise to Nijenhuis operators. This also explains why we chose the terminology 'preHamiltonian' instead of 'integrable' for such operators. These operators naturally appear in the description of the invariant evolutions of curvature flows [35].

Definition 9. A difference operator $R$ is Nijenhuis if

$$
[R a, R b]-R[R a, b]-R[a, R b]+R^{2}[a, b]=0 \quad \text { for all } \quad a, b \in \mathrm{F} .
$$

Clearly, a Nijenhuis operator is also preHamiltonian with

$$
\omega_{R}(a, b)=(R b)_{*}[a]-(R a)_{*}[b]-R[a, b] .
$$


For a rational operator $R=A B^{-1}$, which is defined on $\operatorname{Im} B$, we define the Nijenhuis identity as

$$
\begin{aligned}
& A_{*}[A a]-\left[(A a)_{*}, A\right]+A B^{-1} A B^{-1}\left(B_{*}[B a]-\left[(B a)_{*}, B\right]\right) \\
& \quad=A B^{-1}\left(B_{*}[A a]+A_{*}[B a]-\left[(A a)_{*}, B\right]-\left[(B a)_{*}, A\right]\right) \text { for all } a \in \mathrm{F},
\end{aligned}
$$

where the bracket denotes the commutator of two difference operators.

Theorem 3. If two difference operators $A$ and $B$ form a preHamiltonian pair, then $R=A B^{-1}$ is Nijenhuis.

Proof. Since $A$ and $B$ are preHamiltonian we can write for all $a \in \mathrm{F}$

$$
\begin{aligned}
& A_{*}[A a]-\left[(A a)_{*}, A\right]=A\left(\omega_{A}(a, \bullet)+(A a)_{*}-a_{*} A\right) ; \\
& B_{*}[B a]-\left[(B a)_{*}, B\right]=B\left(\omega_{B}(a, \bullet)+(B a)_{*}-a_{*} B\right) .
\end{aligned}
$$

Hence, we see that, provided that $A$ and $B$ are preHamiltonians, (24) is equivalent to

$$
\begin{gathered}
A B^{-1}\left(B \omega_{A}(a, \bullet)+A \omega_{B}(a, \bullet)-B_{*}[A a]-A_{*}[B a]\right. \\
\left.+(A a)_{*} B+(B a)_{*} A-A a_{*} B-B a_{*} A\right)=0,
\end{gathered}
$$

where the expression inside the parentheses is nothing else than (22). Therefore, given two preHamiltonians difference operators $A$ and $B$, the ratio $A B^{-1}$ is Nijenhuis if and only if $A$ and $B$ form a preHamiltonian pair.

Conversely, we have the following statement:

Theorem 4. Let $R$ be a Nijenhuis rational difference operator with minimal decomposition $A B^{-1}$ such that $B$ is preHamiltonian. Then $A$ and $B$ form a preHamiltonian pair.

Proof. Since $B$ is preHamiltonian, we have for all $a \in \mathrm{F}$

$$
B_{*}[B a]-\left[(B a)_{*}, B\right]=B\left(\omega_{B}(a, \bullet)+(B a)_{*}-a_{*} B\right)
$$

Therefore, we can transform (24) into the equivalent form

$$
\begin{aligned}
A_{*}[A a]-(A a)_{*} A+A a_{*} A \\
\quad=A B^{-1}\left(B_{*}[A a]+A_{*}[B a]+B a_{*} A\right. \\
\left.\quad+A a_{*} B-(B a)_{*} A-(A a)_{*} B-A \omega_{B}(a, \bullet)\right) .
\end{aligned}
$$

Let $C A=D B$ be the left least common multiple of the pair $A$ and $B$. It is also the right least common multiple of the pair $C$ and $D$ since $A B^{-1}$ is minimal. By Lemma 5 (i) there exists a difference operator $\omega_{A}(a, \bullet)$ and thus 2-form $\omega_{A}$ on $\mathrm{F}$ such that

$$
\begin{aligned}
& A_{*}[A a]-(A a)_{*} A+A a_{*} A=A \omega_{A}(a, \bullet) \\
& B_{*}[A a]+A_{*}[B a]+B a_{*} A+A a_{*} B-(B a)_{*} A-(A a)_{*} B-A \omega_{B}(a, \bullet) \\
& \quad=B \omega_{A}(a, \bullet),
\end{aligned}
$$

which implies that $A$ and $B$ form a preHamiltonian pair. 
There is a simple algorithm to determine whether a given difference operator is preHamiltonian and to find the corresponding 2-form $\omega$. Theorem 3 provides an efficient method to check the Nijenhuis property for rational operators, which is important in the theory of integrability.

Example 2. The operators $A$ and $B$ defined in (2) form a preHamiltonian pair. Thus the recursion operator for the Volterra chain (1) is Nijenhuis.

Proof. Let $C=A+\lambda B$. According to Definition 8, we check the existence of a 2-form $\omega_{C}$ in (20). By direct computation, we have

$$
\begin{aligned}
& C_{*}[C a](b)-C_{*}[C b](a) \\
& =u\left\langle u_{1} u_{2} a_{3} b_{2}+\left(u_{1}+u\right) u_{1} a_{2} b_{1}+\left(u+u_{-1}\right) u_{-1} a_{-1} b+u_{-2} u_{-1} a_{-2} b_{-1}\right. \\
& \left.\quad+\lambda u_{1} a_{2} b_{1}+\lambda u_{-1} a_{-1} b\right\rangle_{\mathcal{P}_{a, b}},
\end{aligned}
$$

where $\mathcal{P}_{a, b}$ stands for anti-symmetrisation with respect to $a_{i}$ 's and $b_{j}$ 's. We can now compute its preimage $\omega_{C}(a, b)$ by comparing its highest order either of $a$ or $b$ and we get

$$
\omega_{C}(a, b)=u\left(a_{1} b-a b_{1}\right)+u_{-1}\left(a b_{-1}-a_{-1} b\right) .
$$

It follows from Theorem 3 that the recursion operator $R=A B^{-1}$ for the Volterra chain (1) is Nijenhuis.

The previous two theorems provide the interrelations between preHamiltonian pairs and Nijenhuis operators. The following theorem (analogous to its differential counterpart in [17]) gives another motivation to the definition of a preHamiltonian pair: it is a necessary condition for a rational operator $R=A B^{-1}$ to 'generate' an infinite commuting hierarchy.

Theorem 5. Let $R$ be a rational operator with minimal decomposition $R=A B^{-1}$. Suppose that there exist $\left(f^{(n)}\right)_{n \geq 0} \in \mathrm{F}$ spanning an infinite dimensional space over $\mathrm{k}$ such that for all $n \geq 0, A\left(f^{(n)}\right)=B\left(f^{(n+1)}\right)$ and such that $\left[B\left(f^{(n)}\right), B\left(f^{(m)}\right)\right]=0$ for all $n, m \geq 0$. Then $A$ and $B$ form a preHamiltonian pair.

Proof. Since $\left[B\left(f^{(m)}\right), B\left(f^{(n)}\right)\right]=0$ for all $m, n \geq 0$ by assumption, we have

$$
\left(B_{*}\left[B\left(f^{(n+1)}\right)\right]-\left(B\left(f^{(n+1)}\right)\right)_{*} B\right)\left(f^{(m)}\right)=B\left(-\left(f^{(m)}\right)_{*}\left[B\left(f^{(n+1)}\right)\right]\right) \quad \forall m, n \geq 0 .
$$

Similarly, replacing $B$ with $A$ we get for all $n, m \geq 0$

$$
\left(A_{*}\left[A\left(f^{(n)}\right)\right]-\left(A\left(f^{(n)}\right)\right)_{*} A\right)\left(f^{(m)}\right)=A\left(-\left(f^{(m)}\right)_{*}\left[A\left(f^{(n)}\right)\right]\right) .
$$

Let $C A=D B$ be the left least common multiple of the pair $A$ and $B$. A non-zero difference operator has a finite dimensional kernel over $\mathrm{k}$, therefore one must have for all $n \geq 0$ that

$$
D\left(B_{*}\left[B\left(f^{(n+1)}\right)\right]-\left(B\left(f^{(n+1)}\right)\right)_{*} B\right)=C\left(A_{*}\left[A\left(f^{(n)}\right)\right]-\left(A\left(f^{(n)}\right)\right)_{*} A\right) .
$$

By minimality of the fraction $A B^{-1}$, we deduce that for all $n \geq 0$ there exists a difference operator $P^{(n)}$ such that

$$
\begin{aligned}
B_{*}\left[B\left(f^{(n+1)}\right)\right]-\left(B\left(f^{(n+1)}\right)\right)_{*} B & =B P^{(n)}, \\
A_{*}\left[A\left(f^{(n)}\right)\right]-\left(A\left(f^{(n)}\right)\right)_{*} A & =A P^{(n)} .
\end{aligned}
$$


For all $f \in \mathrm{F}$ we can write $B(f)_{*}=B f_{*}+\left(D_{B}\right)_{f}$, where $\left(D_{B}\right)_{f}$ is defined by $\left(D_{B}\right)_{f}(g)=B_{*}[g](f)$ for all $g \in$ F. $D_{B}$ is a bidifference operator, i.e., $\left(D_{B}\right)_{f}$ is a difference operator and its coefficients are difference operators applied to $f$. In other words $\left(D_{B}\right)_{f}=P_{M}(f) S^{M}+\cdots P_{N}(f) S^{N}$ for all $f$, where $P_{M}, \ldots P_{N}$ are difference operators. We can find a unique pair of bidifference operators $Q$ and $L$ such that $\operatorname{Ord} L_{f}<\operatorname{Ord} B$ for all $f$ and

$$
B_{*}[B(f)]-\left(D_{B}\right)_{f} B=B Q_{f}+L_{f} .
$$

From (33) we see that $L_{f^{(n)}}=0$ for all $n \geq 1$. This implies that $L=0$ since the $f^{(n)}$ span an infinite dimensional space over $\mathrm{k}$. Therefore, for all $f, g$, we have

$$
B_{*}[B(f)](g)-B_{*}[B(g)](f)=B Q_{f}(g)
$$

implying that $B$ is preHamiltonian. Finally, since for all constant $\lambda$, operator

$$
R+\lambda=(A+\lambda B) B^{-1}
$$

satisfies the same hypothesis as $R$, we conclude that $A+\lambda B$ is preHamiltonian.

\section{Towards Applications to Differential-Difference Equations}

In this section we introduce some basic concepts for differential-difference equations relevant to the contents of this paper. More details on the variational difference complex and Lie derivatives can be found in $[15,36]$.

Let $\mathbf{u}=\left(u^{1}(n, t), \ldots, u^{N}(n, t)\right)$ be a vector function of a discrete variable $n \in \mathbb{Z}$ and time variable $t$, where $n$ and $t$ are "independent variables" and $\mathbf{u}$ will play the role of a "dependent" variable in an evolutionary differential-difference system

$$
\mathbf{u}_{t}=\mathbf{f}\left(\mathbf{u}_{p}, \ldots, \mathbf{u}_{q}\right), \quad p \leq q, \quad p, q \in \mathbb{Z}
$$

The Eq. (35) is an abbreviated form to encode the infinite sequence of ordinary differential systems of equations

$$
\partial_{t} \mathbf{u}(n, t)=\mathbf{f}(\mathbf{u}(n+p, t), \ldots, \mathbf{u}(n+q, t)), \quad n \in \mathbb{Z} .
$$

A vector function $\mathbf{f}$ is assumed to be a locally holomorphic function in its arguments. In the majority of cases it will be a rational or polynomial function which does not depend explicitly on the variables $n, t$. The corresponding vector field coincides with (4). Thus there is a bijection between evolutionary derivations of $\mathrm{F}$ and differential-difference systems with $\mathbf{f} \in \mathrm{F}^{N}$.

Definition 10. There are three equivalent definitions of symmetry of an evolutionary equation. We say that $\mathbf{g} \in \mathrm{F}^{N}$ is a symmetry of (35) if

1. $[\mathbf{g}, \mathbf{f}]=0$.

2. $\hat{\mathbf{u}}_{k}=\mathbf{u}_{k}+\epsilon \mathbf{g}_{k}$ satisfy equation (35) $\bmod \epsilon^{2}$ whenever $\mathbf{u}$ is a solution.

3. Equation $\mathbf{u}_{\tau}=\mathbf{g}$ is compatible with (35). 
Symmetries of an equation form a Lie subalgebra in Der F. The existence of an infinite dimensional commutative Lie algebra of symmetries is a characteristic property of an integrable equation and it can be taken as a definition of integrability.

Often the symmetries of integrable equations can be generated by recursion operators [2]. Roughly speaking, a recursion operator is a linear operator $R: \mathrm{F}^{N} \rightarrow \mathrm{F}^{N}$ mapping a symmetry to a new symmetry. For an evolutionary Eq. (35), it satisfies

$$
R_{t}=R_{*}[\mathbf{f}]=\left[\mathbf{f}_{*}, R\right] .
$$

Recursion operators for nonlinear integrable equations are often Nijenhuis operators. Therefore, if the Nijenhuis operator $R$ is a recursion operator of (35), the operator $R$ is also a recursion operator for each of the evolutionary equations in the hierarchy $\mathbf{u}_{t}=R^{k}(\mathbf{f})$, where $k=0,1,2, \ldots$.

Nijenhuis operators are closely related to Hamiltonian and symplectic operators. The general framework in the context of difference variational complex and Lie derivatives can be found in $[15,36]$. Here we recall the basic definitions related to Hamiltonian systems.

For any element $a \in \mathrm{F}$, we define an equivalent class (or a functional) $\int a$ by saying that two elements $a, b \in \mathrm{F}$ are equivalent if $a-b \in \operatorname{Im}(\mathcal{S}-1)$. The space of functionals is denoted by $\mathrm{F}^{\prime}$.

For any functional $\int f \in \mathrm{F}^{\prime}$ (simply written $f \in \mathrm{F}^{\prime}$ without confusion), we define its difference variational derivative (Euler operator) denoted by $\delta_{\mathbf{u}} f \in \mathrm{F}^{N}$ (here we identify the dual space with itself) as

$$
\delta_{\mathbf{u}} f=\left(\delta_{u^{1}} f, \ldots, \delta_{u^{N}} f\right)^{t r}, \quad \delta_{u^{l}} f=\sum_{i \in \mathbb{Z}} \mathcal{S}^{-i} \frac{\partial f}{\partial u_{i}^{l}}=\frac{\partial}{\partial u^{l}}\left(\sum_{i \in \mathbb{Z}} \mathcal{S}^{-i} f\right) .
$$

Definition 11. An evolutionary Eq. (35) is said to be a Hamiltonian equation if there exists a Hamiltonian operator $H$ and a Hamiltonian $\int g \in \mathrm{F}^{\prime}$ such that $\mathbf{u}_{t}=H \delta_{\mathbf{u}} \int g$.

This is the same to say that the evolutionary vector field $\mathbf{f}$ is a Hamiltonian vector field and thus the Hamiltonian operator is invariant along it, that is,

$$
H_{t}=H_{*}[\mathbf{f}]=\mathbf{f}_{*} H+H \mathbf{f}_{*}^{\dagger} .
$$

Nijenhuis recursion operators for some integrable difference equations, e.g., the Narita-Itoh-Bogoyavlensky lattice [37], are no longer weakly nonlocal, but rational difference operators of the form $R=A B^{-1}$. The following statement tells us how operators $A$ and $B$ are related to a given equation.

Theorem 6. If a rational difference operator $R$ with minimal decomposition $A B^{-1}$ is a recursion operator for Eq. (35), then there exists a difference operator $P$ such that

$$
A_{*}[\mathbf{f}]=\mathbf{f}_{*} A+A P, \quad B_{*}[\mathbf{f}]=\mathbf{f}_{*} B+B P .
$$

Proof. To say that $A B^{-1}$ is a minimal decomposition of $R$ means that $A$ and $B$ are right coprime. Let $C$ and $D$ be two left coprime matrix operators with $C$ regular such that $C A=D B$. Such a pair exists by Lemma 5 . Since $R=A B^{-1}$ is a recursion operator of (35), substituting it into (36) we have

$$
R_{t}=R_{*}[\mathbf{f}]=A_{*}[\mathbf{f}] B^{-1}-A B^{-1} B_{*}[\mathbf{f}] B^{-1}=\mathbf{f}_{*} A B^{-1}-A B^{-1} \mathbf{f}_{*},
$$


that is,

$$
\left(A_{*}[\mathbf{f}]-\mathbf{f}_{*} A\right)=A B^{-1}\left(B_{*}[\mathbf{f}]-\mathbf{f}_{*} B\right) .
$$

We rewrite (39) as

$$
C\left(A_{*}[\mathbf{f}]-\mathbf{f}_{*} A\right)=D\left(B_{*}[\mathbf{f}]-\mathbf{f}_{*} B\right) .
$$

By Lemma 5 there exists an operator $P$ such that

$$
A_{*}[\mathbf{f}]-\mathbf{f}_{*} A=A P, \quad B_{*}[\mathbf{f}]-\mathbf{f}_{*} B=B P .
$$

Thus the operators $A$ and $B$ satisfy the same relation (38).

Comparing to (37), for Hamiltonian operators, we have $P=\mathbf{f}_{*}^{\dagger}$. Conversely, it can be easy to show that

Proposition 10. For an Eq. (35) if there exist two operators $A$ and B satisfying (38), then $R=A B^{-1}$ is a recursion operator for the equation.

Proof. By direct computation, we have

$$
\begin{aligned}
R_{t} & =A_{*}[\mathbf{f}] B^{-1}-A B^{-1} B_{*}[\mathbf{f}] B^{-1} \\
& =\left(\mathbf{f}_{*} A+A P\right) B^{-1}-A B^{-1}\left(\mathbf{f}_{*} B+B P\right) B^{-1}=\mathbf{f}_{*} R-R \mathbf{f}_{*}
\end{aligned}
$$

satisfying (36). Thus $R=A B^{-1}$ is a recursion operator.

This proposition has been used in [38] in constructing recursion operators for integrable noncommutative ODEs.

Example 3. For the operators $A$ and $B$ defined in (2) of the Volterra chain (1), the difference operator $P$ in Theorem 6 is $P=\left(1+\mathcal{S}^{-1}\right) u(1-\mathcal{S})$.

In what follows, we give the conditions for a rational recursion operator $R=A B^{-1}$ to generate infinitely many local commuting symmetries. We first prove the following lemma:

Lemma 3. Assume that $B$ is a preHamiltonian operator $R=A B^{-1}$ with minimal decomposition is a recursion operator for $\mathbf{u}_{t}=B(\mathbf{g})$, where $\mathbf{g} \in \mathrm{F}^{N}$. Then $[B(\mathbf{g}), A(\mathbf{g})]=$ 0 .

In particular, if there exists $\mathbf{h} \in \mathrm{F}^{N}$ such that $R$ is a recursion operator for $\mathbf{u}_{t}=B(\mathbf{h})$ and $[B(\mathbf{g}), A(\mathbf{h})]=0$, then $[A(\mathbf{g}), B(\mathbf{h})]=0$.

Proof. We know that $B$ is preHamiltonian. So for any $\mathbf{a} \in \mathrm{F}^{N}$, we have

$$
B_{*}[B \mathbf{a}]-(B \mathbf{a})_{*} B=B\left(\omega_{B}(\mathbf{a}, \bullet)-\mathbf{a}_{*} B\right) .
$$

From Theorem 6, it follows, when $\mathbf{a}=\mathbf{g}$ or $\mathbf{a}=\mathbf{h}$, that

$$
A_{*}[B \mathbf{a}]-(B \mathbf{a})_{*} A=A\left(\omega_{B}(\mathbf{a}, \bullet)-\mathbf{a}_{*} B\right) .
$$

Using (41) for $\mathbf{a}=\mathbf{g}$, we get

$$
[B(\mathbf{g}), A(\mathbf{g})]=A_{*}[B(\mathbf{g})](\mathbf{g})+A \mathbf{g}_{*}[B(\mathbf{g})]-(B \mathbf{g})_{*}[A \mathbf{g}]=A\left(\omega_{B}(\mathbf{g}, \mathbf{g})\right)=0 .
$$

If there exists $\mathbf{h} \in \mathrm{F}^{N}$ such that $R$ is a recursion operator for $\mathbf{u}_{t}=B(\mathbf{h})$ then from the former we deduce that

$$
[B(\mathbf{g}+\mathbf{h}), A(\mathbf{g}+\mathbf{h})]=[B(\mathbf{g}), A(\mathbf{g})]=[B(\mathbf{h}), A(\mathbf{h})]=0 .
$$

Hence $[B(\mathbf{h}), A(\mathbf{g})]=-[B(\mathbf{g}), A(\mathbf{h})]$. 
Proposition 11. Assume that $A$ and $B$ form a preHamiltonian pair and $R=A B^{-1}$ is a recursion operator for $\mathbf{u}_{t}=B\left(\mathbf{g}^{(0)}\right)$, where $\mathbf{g}^{(0)} \in \mathrm{F}^{N}$. If there exists $\mathbf{g}^{(n)} \in \mathrm{F}^{N}$ such that $A\left(\mathbf{g}^{(n)}\right)=B\left(\mathbf{g}^{(n+1)}\right)$ for all $n \geq 0$, then $\left[B\left(\mathbf{g}^{(n)}\right), B\left(\mathbf{g}^{(m)}\right)\right]=0$ for all $n, m \geq 0$.

Proof. We can assume that $A B^{-1}$ is a minimal decomposition of $R$. Indeed, if not we write $A=A_{0} C$ and $B=B_{0} C$ where $R=A_{0} B_{0}^{-1}$ is minimal and replace $\mathbf{g}^{(n)}$ by $C\left(\mathbf{g}^{(n)}\right)$. By Theorem 3, we know that $R$ is Nijenhuis and thus it is a recursion operator for all $B\left(\mathbf{g}^{(n)}\right), n \geq 0$. We proceed the proof by induction on $|n-m|$. If $n=m$ there is nothing to prove. If $|n-m|=1$, we deduce $\left[B\left(\mathbf{g}^{(n)}\right), B\left(\mathbf{g}^{(n+1)}\right)\right]=0$ as a direct application of Lemma 3 since $B\left(\mathbf{g}^{(n+1)}\right)=A\left(\mathbf{g}^{(n)}\right)$ for all $n \geq 0$. Suppose that $\left[B\left(\mathbf{g}^{(n)}\right), B\left(\mathbf{g}^{(m)}\right)\right]=0$ for all $n, m \geq 0$ such that $|n-m| \leq N$, which implies $\left[B\left(\mathbf{g}^{(n+N)}, B\left(\mathbf{g}^{(n+1)}\right)\right]=0\right.$. Hence by Lemma 3, we have $\left[B\left(\mathbf{g}^{(n+N+1)}, B\left(\mathbf{g}^{(n)}\right)\right]=0\right.$.

\section{Rational Recursion Operator for Adler-Postnikov Equation}

In this section, we construct a recursion operator of system

$$
u_{t}=u^{2}\left(u_{2} u_{1}-u_{-1} u_{-2}\right)-u\left(u_{1}-u_{-1}\right):=f
$$

from its Lax representation and show that it is Nijenhuis and generates local commuting symmetries. In general, it is not easy to construct a recursion operator for a given integrable equation although the explicit formula is given. The difficulty lies in how to determine the starting terms of $R$, i.e., the order of the operator, and how to construct its nonlocal terms. Many papers are devoted to this subject, see $[5,39,40]$. If the Lax representation of the equation is known, there is an amazingly simple approach to construct a recursion operator proposed in [41]. The idea in [41] can be developed for the Lax pairs that are invariant under the reduction groups, which applies for both differential and differential-difference equations [7,37].

The Eq. (43) first appeared in [18], where the authors presented its scalar Lax representation. We rewrite it in the matrix form as follows:

$$
\begin{aligned}
& L=\mathcal{S}-\mathbf{U}(\lambda)=\mathcal{S}-\lambda \mathbf{U}^{(1)}-\mathbf{U}^{(0)}=\mathcal{S}-\left(\begin{array}{ccc}
0 & 1 & 0 \\
0 & 0 & 1 \\
\lambda-\frac{1}{u} & \frac{\lambda}{u}
\end{array}\right) \\
& M=D_{t}-\mathbf{V}(\lambda)=D_{t}+\left(\begin{array}{ccc}
-\frac{1}{\lambda^{2}}+u_{-1} & \frac{1}{\lambda}\left(1-u_{-1} u_{-2}\right) & u_{-1} u_{-2}-\frac{u_{-1}}{\lambda^{2}} \\
\lambda u u_{-1}-\frac{u}{\lambda} & u-u_{-1} & \lambda u_{-1}-\frac{u u_{-1}}{\lambda} \\
\lambda^{2} u-u u_{1} & \lambda\left(u u_{1}-1\right) & \lambda^{2}-u
\end{array}\right),
\end{aligned}
$$

where $\lambda$ is a spectral parameter. The commutativity of the above operators leads to the zero curvature condition

$$
\mathbf{U}(\lambda)_{t}=\mathcal{S} \mathbf{V}(\lambda) \mathcal{S}^{-1} \mathbf{U}(\lambda)-\mathbf{U}(\lambda) \mathbf{V}(\lambda)
$$

and subsequently it leads to the system (43). The system (43) defines a derivation $X_{f} \in$ $\mathcal{A}_{1}$ of $\mathcal{R}$ with $f=u^{2}\left(u_{2} u_{1}-u_{-1} u_{-2}\right)-u\left(u_{1}-u_{-1}\right)$. The representation (44), (45) is invariant with respect to the transformations:

$$
\mathcal{S} \mathbf{V}(\lambda) \mathcal{S}^{-1}=-J \mathcal{T} \mathbf{V}\left(\lambda^{-1}\right) \mathcal{T} J, \quad \mathbf{U}^{-1}(\lambda)=J \mathcal{T} \mathbf{U}\left(\lambda^{-1}\right) \mathcal{T} J
$$


and

$$
\mathbf{V}(\lambda)=H \mathbf{V}(-\lambda) H, \quad \mathbf{U}(\lambda)=-H \mathbf{U}(-\lambda) H,
$$

where

$$
J=\left(\begin{array}{lll}
0 & 0 & 1 \\
0 & 1 & 0 \\
1 & 0 & 0
\end{array}\right), \quad H=\left(\begin{array}{rrr}
1 & 0 & 0 \\
0 & -1 & 0 \\
0 & 0 & 1
\end{array}\right) .
$$

The transformation (47) reflects the symmetry $\mathcal{T}(f)=-f$ of the Eq. (43).

For a given matrix $\mathbf{U}$, we can build up a hierarchy of nonlinear systems by choosing different matrices $\mathbf{V}$ with the degree of $\lambda$ from $-2 l$ to $2 l$. The way to construct a recursion operator directly from a Lax representation is to relate the different operators $\mathbf{V}$ using ansatz

$$
\overline{\mathbf{V}}=\left(\lambda^{-2}+\lambda^{2}\right) \mathbf{V}+\mathbf{W}
$$

and then to find the relation between the two flows corresponding to $\overline{\mathbf{V}}$ and $\mathbf{V}$. The multiplier $\lambda^{-2}+\lambda^{2}$ is the automorphic function of the group generated by the transformations $\lambda \mapsto \lambda^{-1}$ and $\lambda \mapsto-\lambda$. Here $\mathbf{W}$ is the remainder and we assume that it has the same symmetry as $\mathbf{V}$ :

$$
\mathbf{W}=\sum_{j=-2}^{2} \lambda^{j} \mathbf{W}^{(j)},
$$

where $\mathbf{W}^{(j)}$ are $3 \times 3$ matrices of the following form [invariant under (48)]

$$
\begin{aligned}
\mathbf{W}^{(2)}=\left(\begin{array}{lll}
a & 0 & b \\
0 & c & 0 \\
d & 0 & e
\end{array}\right) ; & \mathbf{W}^{(0)}=\left(\begin{array}{ccc}
a_{0} & 0 & b_{0} \\
0 & c_{0} & 0 \\
d_{0} & 0 & e_{0}
\end{array}\right) ; \quad \mathbf{W}^{(-2)}=\left(\begin{array}{ccc}
a_{-} & 0 & b_{-} \\
0 & c_{-} & 0 \\
d_{-} & 0 & e_{-}
\end{array}\right) ; \\
\mathbf{W}^{(1)}=\left(\begin{array}{ccc}
0 & r & 0 \\
s & 0 & p \\
0 & q & 0
\end{array}\right) ; & \mathbf{W}^{(-1)}=\left(\begin{array}{ccc}
0 & r_{-} & 0 \\
s_{-} & 0 & p_{-} \\
0 & q_{-} & 0
\end{array}\right)
\end{aligned}
$$

and since $\mathbf{W}$ is invariant under (47), they satisfy

$$
\begin{aligned}
& \mathcal{S}\left(\mathbf{W}^{(2)}\right)=-J \mathcal{T}\left(\mathbf{W}^{(-2)}\right) J ; \quad \mathcal{S}\left(\mathbf{W}^{(1)}\right)=-J \mathcal{T}\left(\mathbf{W}^{(-1)}\right) J ; \\
& \mathcal{S}\left(\mathbf{W}^{(0)}\right)=-J \mathcal{T}\left(\mathbf{W}^{(0)}\right) J .
\end{aligned}
$$

The zero curvature condition leads to

$$
\mathbf{U}_{\tau}=\left(\frac{1}{\lambda^{2}}+\lambda^{2}\right) \mathbf{U}_{t}+\mathcal{S}(\mathbf{W}) \mathbf{U}-\mathbf{U W} .
$$

Substituting the ansatz (49) into (50) and collecting the coefficient of powers of $\lambda$, we obtain six matrix equations for $\mathbf{W}^{(j)}, j=-2, \ldots, 2$. For example, the equation corresponding to linear terms of $\lambda$ is

$$
\mathbf{U}_{\tau}^{(1)}=\mathcal{S}\left(\mathbf{W}^{(1)}\right) \mathbf{U}^{(0)}+\mathcal{S}\left(\mathbf{W}^{(0)}\right) \mathbf{U}^{(1)}-\mathbf{U}^{(1)} \mathbf{W}^{(0)}-\mathbf{U}^{(0)} \mathbf{W}^{(1)} .
$$


Through them we are able to determine the entries of matrices $\mathbf{W}^{(j)}$ and we finally get

$$
\begin{aligned}
& c_{-}=\left(\mathcal{S}^{2}-1\right)^{-1} \frac{u_{t}}{u} \\
& b_{0}=\mathcal{S}^{-1} u\left(\mathcal{S} u-u \mathcal{S}^{-1}\right)^{-1}\left(u\left(\mathcal{S}-\mathcal{S}^{-2}\right) u\left(\mathcal{S}^{2}+\mathcal{S}+1\right)+\mathcal{S}^{2}-\mathcal{S}\right) c_{-} \\
& e_{0}=\left(\mathcal{S}^{2}+\mathcal{S}+1\right)^{-1}\left(-\left(\mathcal{S} \frac{1}{u} \mathcal{S}+\frac{1}{u} \mathcal{S}+\mathcal{S} \frac{1}{u}\right) \mathcal{S} b_{0}+(\mathcal{S}+1) \frac{1}{u}\left(\mathcal{S}^{2}-\mathcal{S}\right) c_{-}\right) \\
& u_{\tau}=u^{2}\left(\mathcal{S}^{3}-1\right) b_{0}-u\left(u \mathcal{S}-\mathcal{S}^{-1} u\right)\left(\mathcal{S}^{2}+\mathcal{S}+1\right) c_{-}+u(\mathcal{S}-1) e_{0}
\end{aligned}
$$

Note that

$$
\mathcal{S} \frac{1}{u} \mathcal{S} u+\frac{1}{u} \mathcal{S} u+\mathcal{S}=(\mathcal{S}+1) \frac{1}{u}\left(\mathcal{S} u-u \mathcal{S}^{-1}\right)+\mathcal{S}^{-1}+1+\mathcal{S}
$$

We simplify the above expression of $e_{0}$. It becomes

$$
\begin{gathered}
e_{0}=\left(\mathcal{S}^{-2}-1\right) u\left(\mathcal{S}^{2}+\mathcal{S}+1\right) c_{-}-\mathcal{S}^{-1}\left(\mathcal{S} u-u \mathcal{S}^{-1}\right)^{-1} \\
\left(u\left(\mathcal{S}-\mathcal{S}^{-2}\right) u\left(\mathcal{S}^{2}+\mathcal{S}+1\right)+\mathcal{S}^{2}-\mathcal{S}\right) c_{-}
\end{gathered}
$$

Substituting $c_{-}, b_{0}$ and $e_{0}$ into (52), we obtain the relation between two symmetry flows $u_{t}$ and $u_{\tau}$. Thus we obtain the following statement:

Proposition 12. A recursion operator for Eq. (43) is

$$
\begin{aligned}
& R=u\left(u\left(\mathcal{S}^{2}-\mathcal{S}^{-1}\right) u+\mathcal{S}^{-1}-1\right)\left(\mathcal{S} u-u \mathcal{S}^{-1}\right)^{-1} \\
& \quad\left(u\left(\mathcal{S}-\mathcal{S}^{-2}\right) u\left(\mathcal{S}^{2}+\mathcal{S}+1\right)+\mathcal{S}^{2}-\mathcal{S}\right)\left(\mathcal{S}^{2}-1\right)^{-1} \frac{1}{u} \\
& +u\left(2 \mathcal{S}^{-1} u-\mathcal{S}^{-2} u-\mathcal{S} u+u-u \mathcal{S}\right)\left(\mathcal{S}^{2}+\mathcal{S}+1\right)\left(\mathcal{S}^{2}-1\right)^{-1} \frac{1}{u}
\end{aligned}
$$

We represent $R$ as

$$
R=R^{(3)}+R^{(1)}+R^{(-1)},
$$

where

$$
\begin{aligned}
& R^{(3)}=u^{2}\left(\mathcal{S}^{3}-1\right) \mathcal{S}^{-1} u\left(\mathcal{S} u-u \mathcal{S}^{-1}\right)^{-1} u\left(\mathcal{S}-\mathcal{S}^{-2}\right) u\left(\mathcal{S}^{2}+\mathcal{S}+1\right)\left(\mathcal{S}^{2}-1\right)^{-1} \frac{1}{u} \\
& R^{(-1)}=u\left(\mathcal{S}^{-1}-1\right)\left(\mathcal{S} u-u \mathcal{S}^{-1}\right)^{-1}\left(\mathcal{S}^{-1}+1\right)^{-1} \frac{1}{u}
\end{aligned}
$$

Note that $R^{(3)}$ is a recursion operator for $u_{t}=u^{2}\left(u_{1} u_{2}-u_{-1} u_{-2}\right)$ [37] and that $R^{(-1)}$ is the inverse recursion operator for the Volterra chain $u_{t}=u\left(u_{1}-u_{-1}\right)$ [20].

The recursion operator (53) is not weakly nonlocal. We now rewrite it as a rational difference operator. It is convenient to first write $R$ as

$$
R=\left(Q \Delta^{-1} C+P\right)\left(\mathcal{S}^{2}-1\right)^{-1} \frac{1}{u}
$$


where

$$
\begin{aligned}
Q & =u\left(u u_{1}-1+\left(1-u u_{-1}\right) S^{-1}\right) ; \quad \Delta=\mathcal{S} u-u \mathcal{S}^{-1} \\
C & =w_{2} \mathcal{S}^{2}-w_{-1} \mathcal{S}, \quad w=1-u_{-1} u_{1} ; \\
P & =\left(u^{2} \mathcal{S} u\left(\mathcal{S}-\mathcal{S}^{-2}\right) u\right. \\
& \left.+Q u_{-1}+u\left(2 \mathcal{S}^{-1} u-\mathcal{S}^{-2} u-\mathcal{S} u+u-u \mathcal{S}\right)\right)\left(\mathcal{S}^{2}+\mathcal{S}+1\right) \\
& +u^{2} \mathcal{S}\left(\mathcal{S}^{2}-\mathcal{S}\right)+Q\left(u_{-1} \mathcal{S}^{-1}+u_{2} \mathcal{S}\right)=\tilde{P}(S+1)+p
\end{aligned}
$$

where $\tilde{P}$ is a difference operator and $p=u\left(u_{2}+2 u_{1}-2 u_{-1}-u_{-2}\right)$.

Lemma 4. The recursion operator $R$ given by (54) can be factorized as $R=A B^{-1}$ with

$$
B=u\left(\mathcal{S}-\mathcal{S}^{-1}\right)\left(\mathcal{S} \alpha+\beta+\mathcal{S}^{-1} \gamma\right)
$$

where

$$
\begin{aligned}
& \alpha=u_{-1} u w_{-1} w-u_{-1} u_{1} w_{1} w_{2} ; \quad \beta=u^{2} w^{2}-u_{-1} u_{1} w_{-2} w_{2} \\
& \gamma=u_{1} u w_{1} w-u_{-1} u_{1} w_{-1} w_{-2} ; \quad w=1-u_{-1} u_{1} .
\end{aligned}
$$

and

$$
A=Q\left(\frac{1}{u} w_{1} \alpha_{1} \mathcal{S}+\frac{1}{u_{1}} w \gamma\right)+P \mathcal{S}^{-1}\left(S \alpha+\beta+S^{-1} \gamma\right)
$$

Proof. To find $A$ and $B$ for (54) we need to rewrite $\Delta^{-1} C$ as a right fraction. It turns out that

$$
C \mathcal{S}^{-1}\left(\mathcal{S} \alpha+\beta+\mathcal{S}^{-1} \gamma\right)=\Delta\left(\frac{1}{u} w_{1} \alpha_{1} \mathcal{S}+\frac{1}{u_{1}} w \gamma\right),
$$

from which we can find that $\alpha, \beta$ and $\gamma$ as stated is a solution. Then $A=R B$ by definition as given in the statement.

The authors in [42] showed that the recursion operators derived from certain Lax representations under certain boundary conditions are Nijenhuis once every step is uniquely determined. Here we prove the Nijenhuis property using the results in Sect. 3.

Theorem 7. The operators $A$ and $B$ defined by (59) and (58) are compatible preHamiltonian operators. In particular, the recursion operator $R$ for Eq. (43) given by (53) is Nijenhuis.

Proof. We know from Lemma 4 that $R=A B^{-1}$. To prove that it is Nijenhuis, we only need to show operators $A$ and $B$ form a preHamiltonian pair following from Theorem 3 .

Let $I=A+\lambda B$. For any $a, b \in \mathrm{F}$ and constant $\lambda$, we use computer algebra package Maple to compute $e^{(0)}=I_{*}[I a](b)-I_{*}[I b](a)$, which is linear in $a$ and its shifts. We take the coefficient of the highest order term $a_{k}$ (here $\left.k=11\right)$ in $e^{(0)}$ and denote it by $v^{(0)}$. Notice that the highest order term in $I$ is $u^{2} u_{1} u_{2} \mathcal{S}^{4} \alpha$. We set $\omega^{(0)}=\frac{1}{\alpha} \mathcal{S}^{-4}\left(\frac{v^{(0)} a_{k}}{u^{2} u_{1} u_{2}}\right)$. We then compute $e^{(1)}=e^{(0)}-I\left(\omega^{(0)}\right)$ and repeat the procedure. Finally we get $e^{(11)}=0$ after $n=11$ steps implying $I$ is preHamiltonian. 
Since the operator $R$ is not weakly nonlocal, the results on the locality of symmetries generated by $R$ in [7] are no longer valid. In the rest of this section, we are going to show that $R$ generates infinitely many commuting symmetries of (43) starting from the equation itself.

Proposition 13. Let $h$ be a difference polynomial such that $R$ is a recursion operator for $u_{t}=h$. Then $h$ lies in the image of $B$. More precisely $h=B(x)$ for some $x \in \mathrm{F}$ and $A(x)$ is a difference polynomial. Moreover, $R$ is a recursion operator for $u_{t}=A(x)$.

We will break the proof of this proposition in two parts using (54). First we will prove that $h=u\left(g_{2}-g\right)$ for some difference polynomial $g$. Second we will show that $C(g)=\Delta(k)$ for some difference polynomial $k$. We begin with proving a few lemmas. To improve the readability, we put them in "Appendix B". We now write the proof for Proposition 13 using these lemmas.

Proof. By Lemma 8, we know that $h=u\left(g_{2}-g\right)$ for some difference polynomial $h$. By Lemmas 9 and 10, for some constant $\lambda \in \mathrm{k}$ we get that

$$
C(g)_{*} u\left(S^{2}-1\right)-C(g)\left(S^{2}-S\right) \equiv \lambda C .
$$

Since $g$ is a difference polynomial, the constant term in $C(g)_{*} u\left(S^{2}-1\right)-C(g)\left(S^{2}-\right.$ $S)-\lambda C$ is $\lambda\left(S-S^{2}\right)$. This constant term must be divisible on the left by $\Delta$, which implies $\lambda=0$. Moreover, we can divide the congruence relation by $\left(S^{2}-S\right)$ on the right since $\Delta$ has a trivial kernel:

$$
C(g)_{*} u\left(1+S^{-1}\right) \equiv C(g) .
$$

After applying Lemma 11 we deduce that $C(g)=\Delta(k)$ for some difference polynomial $k$.

Let $M$ be a generator of the right ideal $C \mathcal{R} \cap \Delta \mathcal{R}$ in $\mathcal{R}$. This means that $M=C E=$ $\triangle D$ for some pair of right coprime difference operators $D$ and $E$. By Lemma 1, there exists $x \in \mathrm{F}$ such that $g=E(x)$ and $k=D(x)$. Since $B=u\left(S^{2}-1\right) E$, we conclude that $h=B(x)$. Finally, $A=Q D+P E$, hence $A(x)=P(g)+Q(k)$ is a difference polynomial. $R$ is a recursion operator for $u_{t}=A(x)$ since $R$ is Nijenhuis following from Theorem 7.

Theorem 8. There exists a sequence $g^{(2)}, g^{(4)}, g^{(6)}, \ldots$ in $\mathrm{F}$ such that

(1) $u^{2}\left(u_{1} u_{2}-u_{-1} u_{-2}\right)-u\left(u_{1}-u_{-1}\right)=B\left(g^{(2)}\right)$;

(2) $A\left(g^{(2 n)}\right)=B\left(g^{(2 n+2)}\right)$ for all $n \geq 1$;

(3) $B\left(g^{(2 n)}\right)$ is a difference polynomial for all $n \geq 1$;

(4) $\left[B\left(g^{(2 n)}\right), B\left(g^{(2 m)}\right)\right]=0$ for all $n, m \geq 1$;

(5) The order of $B\left(g^{(2 n)}\right)$ is $(-2 n, 2 n)$;

(6) $R$ is a recursion operator for all the $u_{t}=B\left(g^{(2 n)}\right)$.

Finally, let $V=\operatorname{Span}_{\mathrm{k}}\left\{B\left(g^{(2 n)}\right) \mid n \geq 1\right\}$. If $f \in \mathrm{F}$ commutes with some element $h \in V$, then $f \in V$.

Proof. We already know that $R$ is a recursion operator for (43), hence by Proposition 13 there exists $g^{(2)} \in \mathrm{F}$ such that statement (1) is satisfied and $A\left(g^{(2)}\right)$ is a difference polynomial. Since $R$ is Nijenhuis (following from Theorem 7) it must be a recursion operator for $u_{t}=A\left(g^{(2)}\right)$ as well. Using Proposition 13 a second time we find $g^{(4)} \in$ F such that $B\left(g^{(4)}\right)=A\left(g^{(2)}\right)$ and $A\left(g^{(4)}\right)$ is a difference polynomial. Iterating this argument we prove the statements (2), (3) and (6). Statement (5) is obvious and statement 
(4) follows from Proposition 11 and Theorem 7. Finally, if $f \in \mathrm{F}$ commutes with $h \in V$, let us sketch the proof of how to show that $f \in V$. If $(M, N)$ is the order of $f$ and $N>0$ it is not hard to prove from the equation

$$
X_{h}\left(f_{*}\right)=\left[h_{*}, f_{*}\right]+X_{f}\left(h_{*}\right) .
$$

Note that the leading term of $f_{*}$ is up to multiplication by a constant the leading term of $B\left(g^{(2 k)}\right)_{*}$ for some $k \geq 2$. Similarly, if $M<0$, one sees that the negative leading term of $f_{*}$ is up to multiplication by a constant the negative leading term of $B\left(g^{(2 l)}\right)_{*}$ for some $l \geq 2$. We conclude by induction on the total order of $f$, after checking that the only $f$ commuting with an element of $V$ and which depend either on $u, \ldots, u_{N}$ or on $u_{-N}, \ldots, 0$ for $N \geq 0$ is $f=0$.

Remark 3. Note that $g^{(2)}=\frac{u_{-1} u u_{1} w_{-1} w w_{1}}{\alpha \gamma}$ is in $\mathrm{F}$ but is not a difference polynomial.

Remark 4. Let $\mathcal{T}$ be the automorphism of $\mathrm{K}$ defined in Sect. 2. Then we have $\mathcal{T} A \mathcal{T}=$ $-A$ and $\mathcal{T} B \mathcal{T}=-B$. This implies that $\mathcal{T}\left(B\left(g^{(2 n)}\right)\right)=-B\left(g^{(2 n)}\right)$ and $\mathcal{T}\left(g^{(2 n)}\right)=g^{(2 n)}$ for all $n \geq 1$.

\section{On Inverse Nijenhuis Recursion Operators}

In [20], the authors listed integrable differential-difference equations with their algebraic properties. For some systems, they presented both recursion operators and their inverse in weakly nonlocal form. In this section, we'll explain the (non)existence of weakly nonlocal inverse recursion operators and how to work out the nonlocal terms based on Theorem 1 and its corollaries in Sect. 2.3 using examples in [20].

We select four examples: in Sect. 6.1, we show the nonexistence of weakly nonlocal inverse recursion operator for the Toda lattice; in Sect. 6.2, we show the existence of weakly nonlocal inverse recursion operator with only one nonlocal term for a relativistic Toda system; in Sect. 6.3, we deal with a recursion operator with two nonlocal terms; for our last example, we demonstrate that the inverse operator $R$ itself is not weakly nonlocal, but that of $R-\mathrm{id}$ is!

\subsection{The Toda lattice. The Toda equation [43] is given by}

$$
q_{t t}=\exp \left(q_{1}-q\right)-\exp \left(q-q_{-1}\right) \text {. }
$$

In the Manakov-Flaschka coordinates [44,45] defined by $u=\exp \left(q_{1}-q\right), v=q_{t}$, it can be rewritten as two-component evolution system:

$$
\left\{\begin{array}{c}
u_{t}=u\left(v_{1}-v\right) \\
v_{t}=u-u_{-1}
\end{array},\right.
$$

which admits two compatible Hamiltonian local structures

$$
H_{1}=\left(\begin{array}{cc}
0 & u(\mathcal{S}-1) \\
\left(1-\mathcal{S}^{-1}\right) u & 0
\end{array}\right), \quad H_{2}=\left(\begin{array}{cc}
u\left(\mathcal{S}-\mathcal{S}^{-1}\right) u & u(\mathcal{S}-1) v \\
v\left(1-\mathcal{S}^{-1}\right) u & u \mathcal{S}-\mathcal{S}^{-1} u
\end{array}\right) .
$$

It is clear to see that $\operatorname{Ord} H_{1}=2$ and that the kernel of $H_{1}$ is spanned by $\left(\begin{array}{l}\frac{1}{u} \\ 0\end{array}\right)$ and $\left(\begin{array}{l}0 \\ 1\end{array}\right)$. One can check that the kernel of $H_{2}$ is spanned by $\left(\begin{array}{l}\frac{1}{u} \\ 0\end{array}\right)$. In other words, $H_{1}$ and $H_{2}$ have 
a common right divisor $C$ of the total order being 1 and can be written as $H_{1}=B C$ and $H_{2}=A C$, where $\operatorname{Ord} B=1$ and $\operatorname{Ord} A=3$, that is,

$$
\begin{aligned}
& H_{1}=B C=\left(\begin{array}{cc}
0 u(1-\mathcal{S}) \\
1 & 0
\end{array}\right)\left(\begin{array}{rr}
\left(1-\mathcal{S}^{-1}\right) u & 0 \\
0 & 1
\end{array}\right) \\
& H_{2}=A C=\left(\begin{array}{cc}
u(\mathcal{S}+1) & u(\mathcal{S}-1) v \\
v & u \mathcal{S}-\mathcal{S}^{-1} u
\end{array}\right)\left(\begin{array}{cc}
\left(1-\mathcal{S}^{-1}\right) u & 0 \\
0 & 1
\end{array}\right) .
\end{aligned}
$$

Thus $B$ has full kernel and $A$ has trivial kernel. Thus the recursion operator

$$
R=H_{2} H_{1}^{-1}=A B^{-1}
$$

is weakly nonlocal but $B A^{-1}$ is not. Indeed,

$$
R=\left(\begin{array}{cc}
v_{1} & u \mathcal{S}+u \\
1+\mathcal{S}^{-1} & v
\end{array}\right)+\left(\begin{array}{c}
u\left(v_{1}-v\right) \\
u-u_{-1}
\end{array}\right)(\mathcal{S}-1)^{-1}\left(\begin{array}{ll}
\frac{1}{u} & 0
\end{array}\right)
$$

6.2. A relativistic Toda system. The relativistic Toda system [46] is given by

$$
q_{t t}=q_{t} q_{-1 t} \frac{\exp \left(q_{-1}-q\right)}{1+\exp \left(q_{-1}-q\right)}-q_{t} q_{1 t} \frac{\exp \left(q-q_{1}\right)}{1+\exp \left(q-q_{1}\right)} .
$$

Introducing the dependent variables as follows [47]:

$$
u=\frac{q_{t} \exp \left(q-q_{1}\right)}{1+\exp \left(q-q_{1}\right)}, \quad v=\frac{q_{t}}{1+\exp \left(q-q_{1}\right)},
$$

then the equation can be written as

$$
\left\{\begin{array}{c}
u_{t}=u\left(u_{-1}-u_{1}+v-v_{1}\right) \\
v_{t}=v\left(u_{-1}-u\right)
\end{array}\right.
$$

It admits two compatible Hamiltonian local structures

$$
H_{1}=\left(\begin{array}{cc}
0 & u(1-\mathcal{S}) \\
\left(\mathcal{S}^{-1}-1\right) u & u \mathcal{S}-\mathcal{S}^{-1} u
\end{array}\right), \quad H_{2}=\left(\begin{array}{cc}
u\left(\mathcal{S}^{-1}-\mathcal{S}\right) u & u(1-\mathcal{S}) v \\
v\left(\mathcal{S}^{-1}-1\right) u & 0
\end{array}\right) .
$$

It is clear to see that $\operatorname{Ord} H_{1}=2$ and that the kernel of $H_{1}$ is spanned by $\left(\begin{array}{l}\frac{1}{u} \\ 0\end{array}\right)$ and $\left(\begin{array}{l}1 \\ 1\end{array}\right)$. Similarly $\operatorname{Ord} H_{2}=2$ and the kernel of $H_{2}$ is spanned by $\left(\begin{array}{c}\frac{1}{u} \\ 0\end{array}\right)$ and $\left(\begin{array}{l}0 \\ \frac{1}{v}\end{array}\right)$. In other words, $H_{1}$ and $H_{2}$ have a common right divisor Ord $C=1$ and can be written as

$$
\begin{aligned}
& H_{1}=B C=\left(\begin{array}{cc}
0 & u(1-\mathcal{S}) \\
1 & u \mathcal{S}-\mathcal{S}^{-1} u
\end{array}\right)\left(\begin{array}{cc}
\left(1-S^{-1}\right) u & 0 \\
0 & 1
\end{array}\right) \\
& H_{2}=A C=\left(\begin{array}{cc}
u(\mathcal{S}+1) u(1-\mathcal{S}) v \\
-v & 0
\end{array}\right)\left(\begin{array}{cc}
\left(1-\mathcal{S}^{-1}\right) u & 0 \\
0 & 1
\end{array}\right),
\end{aligned}
$$


where $A$ and $B$ are of the total order 1 and their kernels are of dimension 1 Therefore both recursion operator $R=A B^{-1}$ and its inverse $R^{-1}=B A^{-1}$ are weakly nonlocal, and

$$
\begin{aligned}
& R=\left(\begin{array}{c}
u \mathcal{S}+u+v_{1}+u_{1}+u \mathcal{S}^{-1} u \mathcal{S}+u \\
v+v \mathcal{S}^{-1} \\
v
\end{array}\right)-\left(\begin{array}{c}
u_{t} \\
v_{t}
\end{array}\right)(\mathcal{S}-1)^{-1}\left(\frac{1}{u} 0\right) \\
& R^{-1}=\left(\begin{array}{cc}
\frac{1}{v_{1}} & -\frac{u}{v_{1}^{2}} \mathcal{S}+\frac{u}{v^{2}}-\frac{2 u}{v v_{1}} \\
-\mathcal{S}^{-1} \frac{1}{v}-\frac{1}{v_{1}} & \frac{u}{v_{1}^{2}} \mathcal{S}+\mathcal{S}^{-1} \frac{u}{v^{2}}+\frac{2 u}{v v_{1}}+\frac{1}{v}
\end{array}\right)+\left(\begin{array}{c}
\frac{u}{v_{1}}-\frac{u}{v} \\
\frac{u_{-1}}{v_{-1}}-\frac{u}{v_{1}}
\end{array}\right)(\mathcal{S}-1)^{-1}\left(\frac{1}{u}-\frac{2}{v}\right)
\end{aligned}
$$

Note that the kernel of $A$ is spanned by $\left(\begin{array}{c}0 \\ \frac{1}{v}\end{array}\right)$, the kernel of $A^{\dagger}$ is spanned by $\left(\begin{array}{c}\frac{1}{u} \\ -\frac{2}{v}\end{array}\right)$ and $B\left(\begin{array}{l}0 \\ \frac{1}{v}\end{array}\right)=\left(\begin{array}{c}\frac{u}{v}-\frac{u}{v_{1}} \\ \frac{u}{v_{1}}-\frac{u_{-1}}{v_{-1}}\end{array}\right)$. This explains the nonlocal term in the inverse of the recursion operator.

6.3. The Ablowitz-Ladik lattice. Consider the Ablowitz-Ladik lattice [19]

$$
\left\{\begin{array}{l}
u_{t}=(1-u v)\left(\alpha u_{1}-\beta u_{-1}\right) \\
v_{t}=(1-u v)\left(\beta v_{1}-\alpha v_{-1}\right)
\end{array}\right.
$$

Its recursion operator [48]

$$
\begin{aligned}
R & =\left(\begin{array}{cc}
(1-u v) \mathcal{S}-u_{1} v-u v_{-1} & -u u_{1} \\
v v_{-1} & (1-u v) \mathcal{S}^{-1}
\end{array}\right)+\left(\begin{array}{c}
-u \\
v
\end{array}\right)(\mathcal{S}-1)^{-1}\left(v_{-1} u_{1}\right) \\
& -\left(\begin{array}{c}
(1-u v) u_{1} \\
-(1-u v) v_{-1}
\end{array}\right)(\mathcal{S}-1)^{-1}\left(\frac{v}{1-u v} \frac{u}{1-u v}\right)
\end{aligned}
$$

can be written as $R=A B^{-1}$, where by letting $w=1-u v, w_{i}=\mathcal{S}^{i} w$ and $p=$ $u_{1} v-u v_{-1}$ we have

$$
A=\left(\begin{array}{cc}
w \mathcal{S}\left(\frac{u v}{v_{1}}-u_{1}\right)+\frac{u_{1} v w}{v_{1}}-\frac{u v_{-1} w_{1}}{v_{1}} & w \mathcal{S} \frac{u}{p}\left(1-\mathcal{S}^{-1}\right)-\frac{u^{2} v_{-1}}{p}+\frac{u u_{1} v}{p} \mathcal{S}^{-1} \\
w \mathcal{S}^{-1}\left(v_{-1}-\frac{v^{2}}{v_{1}}\right)-u_{1} v v_{-1}+\frac{u v^{2} v_{-1}}{v_{1}}-w \mathcal{S}^{-1} \frac{v}{p}\left(1-\mathcal{S}^{-1}\right)+\frac{u v v_{-1}}{p}-\frac{u_{1} v^{2}}{p} \mathcal{S}^{-1}
\end{array}\right)
$$

and

$$
B=\left(\begin{array}{cc}
\frac{w}{v} \mathcal{S}^{-1} p-u_{1} w+\frac{u v w_{1}}{v_{1}} & \frac{u}{p}\left(1-\mathcal{S}^{-1}\right) \\
v_{-1} w-\frac{v^{2} w_{1}}{v_{1}} & -\frac{v}{p}\left(1-\mathcal{S}^{-1}\right)
\end{array}\right)
$$

The operator $A$ can be factorized as follows:

$$
\begin{aligned}
& \left(\begin{array}{c}
1 \\
\left(v_{-2} v-v_{-1}^{2}\right) w r \mathcal{S}^{-1} \\
r
\end{array}\right) \\
& \left(\begin{array}{c}
\left.\frac{q\left(u_{1} v w-u v_{-1} w_{1}\right)}{\left(u v-u_{1} v_{1}\right)}-\frac{w}{\left(v_{-1} v_{1} w_{1}-v^{2} w\right)} \mathcal{S}-\frac{u_{1} v w-u v_{-1} w_{1}}{\left(u v-u_{1} v_{1}\right)\left(v_{-2} v w-v_{-1}^{2} w_{-1}\right)}\right) D, \\
0
\end{array}\right) D
\end{aligned}
$$


where

$$
\begin{gathered}
r=\frac{1}{u v_{-1} w_{-1}-u_{-1} v_{-2} w} ; \quad q=\frac{1}{\left(v_{-1} v_{1} w_{1}-v^{2} w\right)\left(v_{-2} v w-v_{-1}^{2} w_{-1}\right)} \\
D=\left(\begin{array}{c}
v_{-1} w\left(v_{-1} v_{1} w_{1}-v^{2} w\right) \mathcal{S}^{-2} \\
-v w\left(v_{-2} v_{1} w_{1}-v_{-1} v w_{-1}\right) \mathcal{S}^{-1} \\
+v_{1} w\left(v_{-2} v w-v_{-1}^{2} w_{-1}\right) \\
0 \\
\frac{1}{v_{1}}\left(u_{1} v_{1}-u v\right)\left(v_{-2} v w-v_{-1}^{2} w_{-1}\right) \begin{array}{c}
v_{-1} w \mathcal{S}^{-2}+\left(u v_{-2} w-u_{1} v_{-1} w_{-1}\right) \frac{v}{p} \mathcal{S}^{-1} \\
+\left(v_{-1}^{2} w_{-1}-v_{-2} v w\right) \frac{u}{p}
\end{array}
\end{array}\right.
\end{gathered}
$$

Note that $\operatorname{Ord} A=\operatorname{Ord} D=2$ and $\operatorname{ker} D=\operatorname{ker} A$, which is spanned by

$$
\mathbf{h}^{(1)}=\left(\begin{array}{c}
-\frac{1}{u_{1} v-u v_{-1}} \\
\frac{v}{v_{1}}
\end{array}\right) \quad \text { and } \quad \mathbf{h}^{(2)}=\left(\begin{array}{c}
\frac{u v_{1}}{u_{1} v-u v_{-1}} \\
u_{1} v_{1}-1
\end{array}\right) .
$$

Thus the operator $A$ is a full kernel operator and hence the inverse of $A B^{-1}$ is weakly nonlocal. Note that ker $D^{\dagger}$ is spanned by

$$
\begin{aligned}
& \mathbf{g}^{(1)}=\left(\begin{array}{c}
v q \\
0
\end{array}\right)=\left(\begin{array}{c}
\frac{v}{\left(v_{-2} v w-v_{-1}^{2} w_{1}\right)\left(v_{-1} v_{1} w_{1}-v^{2} w\right)} \\
0
\end{array}\right) \text { and } \\
& \mathbf{g}^{(2)}=\left(\begin{array}{c}
-\frac{v_{-1} q}{w} \\
0
\end{array}\right) .
\end{aligned}
$$

Thus $\operatorname{ker} A^{\dagger}$ is spanned by

$$
\begin{aligned}
& \left(\begin{array}{ll}
1 & \mathcal{S} w\left(v_{-1}^{2}-v_{-2} v\right) \\
0 & \frac{1}{r}
\end{array}\right)\left(\begin{array}{cc}
\frac{\left(u v-u_{1} v_{1}\right)}{q\left(u_{1} v w-u v_{-1} w_{1}\right)} & 0 \\
\mathcal{S}^{-1} \frac{w\left(u v-u_{1} v_{1}\right)\left(v_{-2} v w-v_{-1}^{2} w_{-1}\right)}{u_{1} v w-u v_{-1} w_{1}}+v_{-1} v_{1} w_{1}-v^{2} w & 1
\end{array}\right) \mathbf{g}^{(1)} \\
& =\left(\begin{array}{c}
v_{1} \\
u_{-1}
\end{array}\right)
\end{aligned}
$$

and similarly $\left(\frac{\frac{v}{1-u v}}{\frac{u}{1-u v}}\right)$. Moreover, we have

$$
B\left(\mathbf{h}^{(1)}\right)=\left(\begin{array}{c}
u \\
-v
\end{array}\right) ; \quad B\left(\mathbf{h}^{(2)}\right)=\left(\begin{array}{c}
(1-u v) u_{-1} \\
-(1-u v) v_{1}
\end{array}\right)
$$

These give us the nonlocal term appearing in the inverse operator as stated in Theorem 1, and indeed

$$
\begin{aligned}
& R^{-1}=\left(\begin{array}{cc}
(1-u v) \mathcal{S}^{-1} & u u_{-1} \\
-v v_{1} & (1-u v) \mathcal{S}-u v_{1}-u_{-1} v
\end{array}\right) \\
& +\left(\begin{array}{c}
u \\
-v
\end{array}\right)(\mathcal{S}-1)^{-1}\left(\begin{array}{ll}
v_{1} u_{-1}
\end{array}\right) \\
& +\left(\begin{array}{c}
(1-u v) u_{-1} \\
-(1-u v) v_{1}
\end{array}\right)(\mathcal{S}-1)^{-1}\left(\frac{v}{1-u v} \frac{u}{1-u v}\right) .
\end{aligned}
$$


6.4. The Kaup-Newell lattice. Consider the Kaup-Newell lattice [49]:

$$
\left\{\begin{array}{c}
u_{t}=a\left(\frac{u_{1}}{1-u_{1} v_{1}}-\frac{u}{1-u v}\right)+b\left(\frac{u}{1+u v_{1}}-\frac{u_{-1}}{1+u_{-1} v}\right) \\
v_{t}=a\left(\frac{v}{1-u v}-\frac{v_{-1}}{1-u_{-1} v_{-1}}\right)+b\left(\frac{v_{1}}{1+u v_{1}}-\frac{v}{1+u_{-1} v}\right)
\end{array}:=a K_{1}+b K_{-1} .\right.
$$

Its recursion operator

$$
\begin{aligned}
R= & \left(\begin{array}{cc}
-\frac{1}{\left(1-u_{1} v_{1}\right)^{2}} \mathcal{S}+\frac{1}{(1-u v)^{2}}-\frac{2 u_{1} v}{\left(1-u_{1} v_{1}\right)(1-u v)}-\frac{u_{1}^{2}}{\left(1-u_{1} v_{1}\right)^{2}} \mathcal{S}+\frac{u^{2}}{(1-u v)^{2}}-\frac{2 u u_{1}}{(1-u v)\left(1-u_{1} v_{1}\right)} \\
-\frac{v_{-1}^{2}}{\left(1-u_{-1} v_{-1}\right)^{2}} \mathcal{S}^{-1}-\frac{v^{2}}{(1-u v)^{2}} & -\frac{1}{\left(1-u_{-1} v_{-1}\right)^{2}} \mathcal{S}^{-1}+\frac{1-2 u v}{(1-u v)^{2}}
\end{array}\right) \\
& -2 K_{1}(\mathcal{S}-1)^{-1}\left(\frac{v}{1-u v} \frac{u}{1-u v}\right),
\end{aligned}
$$

can be written as $R=A B^{-1}$, where

$$
\begin{aligned}
A & =\left(\begin{array}{rr}
(\mathcal{S}-1) \frac{1}{v(1-u v)}\left(1-\mathcal{S}^{-1}\right)+2(\mathcal{S}-1) \frac{u}{1-u v} \mathcal{S}^{-1} & (\mathcal{S}-1) \frac{u}{1-u v} \\
\left(1-\mathcal{S}^{-1}\right) \frac{v}{1-u v}\left(\mathcal{S}^{-1}+1\right) & \left(1-\mathcal{S}^{-1}\right) \frac{v}{1-u v}
\end{array}\right) \\
& =\left(\begin{array}{cc}
\mathcal{S}-1 & 0 \\
0 & 1-\mathcal{S}^{-1}
\end{array}\right)\left(\begin{array}{cc}
\frac{u}{1-u v} & 0 \\
0 & \frac{v}{1-u v}
\end{array}\right)\left(\begin{array}{cc}
2-\frac{1}{u v} & 1 \\
1 & 1
\end{array}\right)\left(\begin{array}{cc}
\mathcal{S}^{-1}-1 & 0 \\
2 & 1
\end{array}\right)
\end{aligned}
$$

and

$$
B=\left(\begin{array}{cc}
\frac{1-u v}{v}\left(\mathcal{S}^{-1}-1\right)-u \\
0 & v
\end{array}\right)
$$

The operator $A$ does not have a full kernel since $\operatorname{Ord} A=3$ and its kernel is spanned by $\left(\begin{array}{c}1 \\ -2\end{array}\right)$. Surprisingly, operator $C=A-B$ can be factorised as follows:

$$
\left(\begin{array}{cc}
1 & \frac{1}{v_{1}^{2}} \mathcal{S} \\
0 & 1
\end{array}\right)\left(\begin{array}{cc}
1 & 0 \\
\left(u v-\mathcal{S}^{-1}\right) \frac{v_{1}^{2}}{1-u^{2} v_{1}^{2}} & 1
\end{array}\right)\left(\begin{array}{cc}
\frac{1+u v_{1}}{v_{1}^{2}(1-u v)} & 0 \\
0 & 1
\end{array}\right) D
$$

where

$$
D=\left(\begin{array}{cc}
\left(v-2 v_{1}+u v v_{1}\right)+v\left(1-u v_{1}\right) \mathcal{S}^{-1} & v\left(1-u^{2} v_{1}^{2}\right) \\
v\left(1-\mathcal{S}^{-1}\right) \frac{1+u v_{1}}{1-u v_{1}} & 0
\end{array}\right)
$$

Note that $\operatorname{Ord} C=\operatorname{Ord} D=1$ and $\operatorname{ker} D=\operatorname{ker} C$, which is spanned by $\mathbf{h}=$ $\left(\begin{array}{c}\frac{1-u v_{1}}{1+u v_{1}} \\ \frac{2 v_{1}}{v\left(1+u v_{1}\right)}-\frac{2}{\left(1+u_{-1} v\right)}\end{array}\right)$. Thus operator $C$ is a full kernel operator and hence the inverse of $(A-B) B^{-1}$ is weakly nonlocal as presented in [20] and it equals to

$$
\begin{aligned}
(R-\mathrm{id})^{-1}= & \left(\begin{array}{cc}
\frac{1}{\left(1+u_{-1} v\right)^{2}} \mathcal{S}^{-1}-\frac{1+2 u v_{1}}{\left(1+u v_{1}\right)^{2}}-\frac{u^{2}}{\left(1+u v_{1}\right)^{2}} \mathcal{S}+\frac{u_{-1}^{2}}{\left(1+u_{-1} v\right)^{2}}-\frac{2 u u_{-1}}{\left(1+u_{-1} v\right)\left(1+u v_{1}\right)} \\
-\frac{v^{2}}{\left(1+u_{-1} v\right)^{2}} \mathcal{S}^{-1}-\frac{v_{1}^{2}}{\left(1+u v_{1}\right)^{2}} \frac{1}{\left(1+u v_{1}\right)^{2}} \mathcal{S}-\frac{1}{\left(1+u_{-1} v\right)^{2}}-\frac{2 u_{-1} v_{1}}{\left(1+u_{-1} v\right)\left(1+u v_{1}\right)}
\end{array}\right) \\
& -2 K_{-1}(\mathcal{S}-1)^{-1}\left(\frac{v_{1}}{1+u v_{1}} \frac{u_{-1}}{1+u_{-1} v}\right) .
\end{aligned}
$$


Note that $\operatorname{ker} D^{\dagger}$ is spanned by $\left(\begin{array}{c}0 \\ \frac{1}{v}\end{array}\right)$ and thus $\operatorname{ker} C^{\dagger}$ is spanned by

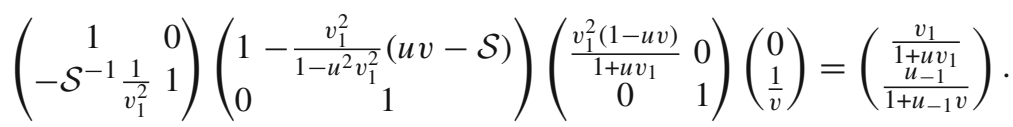

Moreover, we have

$$
B(\mathbf{h})=2\left(\frac{\frac{u}{1+u v_{1}}-\frac{u_{-1}}{1+u_{-1} v}}{\frac{v}{1+u v_{1}}-\frac{v_{-1} v}{1+u_{-1} v}}\right)=2 K_{-1}
$$

These give us the nonlocal term appearing in the inverse operator as shown in (63).

\section{Conclusions}

In this paper we have built a rigorous algebraic setting for difference and rational (pseudo-difference) operators with coefficients in a difference field $\mathrm{F}$ and study their properties. In particular, we formulate a criteria for a rational operator to be weakly nonlocal. We have defined and studied preHamiltonian pairs, which is a generalization of the well known bi-Hamiltonian structures in the theory of integrable systems. By definition a preHamiltonian operator is an operator whose images form a Lie subalgebra in the Lie algebra of evolutionary derivations of F. The latter can be directly verified and it is a relatively simple problem comparing to the verification of the Jacobi identity for Hamiltonian operators. We have shown that a recursion Nijenhuis operator is a ratio of difference operators from a preHamiltonian pair. Thus for a given rational operator, to test whether it is Nijenhuis or not can be done systematically. We applied our theoretical results to integrable differential difference equations in two aspects:

- We have constructed a rational recursion operator $R(53)$ for Adler-Postnikov integrable Eq. (43) and shown that it can be written as the ratio of a preHamiltonian pair and thus it is Nijenhuis. Moreover, we proved that $R$ produces infinitely many commuting local symmetries;

- For a given recursion operator we can answer the question whether the inverse operator is weakly nonlocal and, if so, how to bring it to the standard weakly nonlocal from (examples in Section 6).

In Sect. 6.4 we show that for a weakly nonlocal recursion operator $R$ which does not have a weakly nonlocal inverse, may exist a constant $\gamma \in \mathrm{k}$ such that $(R-\gamma \mathrm{id})^{-1}$ is weakly nonlocal. In other words, the total order of the difference operator $A-\gamma B$ in the factorisation $R=A B^{-1}$ may be lower for a certain choice of $\gamma$. This observation requires further investigation.

The concept of preHamiltonian operators deserves further attention. These operators naturally appear in the description of the invariant evolutions of curvature flows in homogeneous spaces in both continuous [50] and discrete [35] setting. In the future, we'll look into the geometric implication of such operators.

In this paper, we mainly explored the relation between PreHamiltonian operators and Nijenhuis operators. We are going to investigate how preHamiltonian pairs relate to biHamiltonian pairs. In our forthcoming paper [34], we'll present the following main result: if $H$ is a Hamiltonian (a priori nonlocal, i.e. rational) operator, then to find a 
second Hamiltonian $K$ compatible with $H$ is the same as to find a preHamiltonian pair $A$ and $B$ such that $A B^{-1} H$ is skew-symmetric.

We have discovered that Adler-Postnikov integrable equation (43) is indeed a Hamiltonian system. This equation can be written as $u_{t}=H \delta_{u}(\ln u)$, where $H$ is the following skew-symmetric rational operator

$$
\begin{aligned}
H & =u^{2} u_{1} u_{2}^{2} \mathcal{S}^{2}-\mathcal{S}^{-2} u^{2} u_{1} u_{2}^{2}+\mathcal{S}^{-1} u u_{1}\left(u+u_{1}\right)-u u_{1}\left(u+u_{1}\right) \mathcal{S} \\
& +u\left(1-\mathcal{S}^{-1}\right)\left(1-u u_{1}\right)\left(\mathcal{S} u-u \mathcal{S}^{-1}\right)^{-1}\left(1-u u_{1}\right)(\mathcal{S}-1) u .
\end{aligned}
$$

In [34], we are going to show that $H$ is a Hamiltonian operator for Eq. (43) and explain how it is related to the recursion operator (53).

Acknowledgements. The paper is supported by AVM's EPSRC grant EP/P012655/1 and JPW's EPSRC grant EP/P012698/1. Both authors gratefully acknowledge the financial support. JPW and SC were partially supported by Research in Pairs grant no. 41670 from the London Mathematical Society; SC also thanks the University of Kent for the hospitality received during his visit in July 2017. SC was supported by a Junior Fellow award from the Simons Foundation. AVM is grateful for a partial support by the Ministry of Education and Science of Russian Federation, project 1.13560.2019/13.1.

Open Access This article is distributed under the terms of the Creative Commons Attribution 4.0 International License (http://creativecommons.org/licenses/by/4.0/), which permits unrestricted use, distribution, and reproduction in any medium, provided you give appropriate credit to the original author(s) and the source, provide a link to the Creative Commons license, and indicate if changes were made.

Publisher's Note Springer Nature remains neutral with regard to jurisdictional claims in published maps and institutional affiliations.

\section{Appendix A. Basic Concepts for a Unital Associative Principal Ideal Ring}

Recall the definitions of some basic concepts for a unital associative ring $\mathfrak{R}$ (see for example [25]).

A left (respectively right) ideal of $\mathfrak{R}$ is an additive subgroup $\mathfrak{I} \subset \mathfrak{R}$ such that $\mathfrak{R} \mathfrak{I}=\mathfrak{I}$ (resp. $\mathfrak{I} \mathfrak{R}=\mathfrak{I})$. $a \Re)$.

A left (resp. right) principal ideals generated by $a \in \mathfrak{R}$ is, by definition, $\mathfrak{R} a$ (resp.

A ring is called a principal ideal ring, if every left and right ideal of the ring is principal. In what follows we assume that the ring $\Re$ is both a left and a right principal ideal ring, meaning that every left ideal of $\mathfrak{R}$ and every right ideal of $\mathfrak{R}$ is principal.

Given an element $a \in \mathfrak{R}$, an element $d$ is called a right (resp. left) divisor of $a$ if $a=b d$ (resp. $a=d b$ ) for some $b \in \mathfrak{R}$. An element $m \in \mathfrak{R}$ is called left (resp. right) multiple of $a$ if $m=b a$ (resp. $m=a b$ ) for some $b \in \mathfrak{R}$.

Given elements $a, b \in \mathfrak{R}$, their right (resp. left) greatest common divisor ( $\mathrm{gcd}$ ) is the generator $d$ of the left (resp. right) ideal generated by $a$ and $b: \mathfrak{R} a+\mathfrak{R} b=\mathfrak{R} d$ (resp. $a \Re+b \Re=d \Re)$. It is uniquely defined up to multiplication by an invertible element. It follows that $d$ is a right (resp. left) divisor of both $a$ and $b$, and we have the Bezout identity $d=u a+v b$ (resp. $d=a u+b v$ ) for some $u, v \in \mathfrak{R}$.

Similarly, the left (resp. right) least common multiple (lcm) of $a$ and $b$ is an element $m \in \mathfrak{R}$ defined uniquely, up to multiplication by an invertible element, as the generator of the intersection of the left (resp. right) principal ideals generated by $a$ and by $b$ : $\mathfrak{R} m=\mathfrak{R} a \cap \mathfrak{R} b$ (resp. $m \mathfrak{R}=a \mathfrak{R} \cap b \mathfrak{R})$.

We say that $a$ and $b$ are right (resp. left) coprime if their right (resp. left) greatest common divisor is 1 (or invertible), namely if the left (resp. right ) ideal that they generate 
is the whole ring $\mathfrak{R} a+\mathfrak{R} b=\mathfrak{R}$ (resp. $a \mathfrak{R}+b \mathfrak{R}=\mathfrak{R}$ ). In particular there exist $u, v \in \mathfrak{R}$ such that $u a+v b=1$ (resp. $a u+b v=1$ ).

An element $a \in \mathfrak{R} \backslash\{0\}$ is called a right zero divisor if there exists $b \in \mathfrak{R} \backslash\{0\}$ (called a left zero divisor) such that $b a=0$.

A non-zero element $a \in \mathfrak{R}$ is called regular if it is neither a left nor a right zero divisor. A set of regular elements $\mathfrak{R}^{\times}=\{a \in \mathfrak{R} \mid a$ is regular $\}$ is a multiplicative monoid of $\mathfrak{R}$.

A ring $\mathfrak{R}$ is called a domain, if it does not have zero divisors.

A domain $\mathfrak{R}$ is called right (left) Euclidean, if there exists a function

$$
\text { Ord : } \mathfrak{R} \backslash\{0\} \mapsto \mathbb{Z}_{\geq 0},
$$

such that

1. Ord $(a) \leq \operatorname{Ord}(a b) \geq \operatorname{Ord}(b), \quad \forall a, b \in \mathfrak{R} \backslash\{0\}$,

2. for any $a, b \in \mathfrak{R}, b \neq 0$ there exist unique $c_{r}, q_{r} \in \mathfrak{R}$ (resp. $c_{l}, q_{l} \in \mathfrak{R}$ ), such that

$$
a=b c_{r}+q_{r}=c_{l} b+q_{l}
$$

and $q_{r}=0$ or $\operatorname{Ord} q_{r}<\operatorname{Ord} b\left(\operatorname{resp} . q_{l}=0\right.$ or $\left.\operatorname{Ord} q_{l}<\operatorname{Ord} b\right)$.

A principal ideal ring $\mathfrak{R}$ satisfies the right (and left) Ore property (Theorem 2.2 (c) in [25]). Namely, for any $a \in \mathfrak{R}, b \in \mathfrak{R}^{\times}$there exist $c \in \mathfrak{R}^{\times}, d \in \mathfrak{R}$ (resp. $c_{1} \in \mathfrak{R}^{\times}, d_{1} \in \mathfrak{R}$ ) such that $a c=b d$ (resp. $\left.c_{1} a=d_{1} b\right)$.

Lemma 5. Let $\Re$ be a principal ideal ring. Let $a$ and $b$ be two right coprime elements in $\mathfrak{R}$ with $b$ regular. Then there exists two left coprime elements $c, d \in \mathfrak{R}$ with c regular such that $c a=d b$. Moreover,

(i) if $c p=d q$ for some $p, q \in \mathfrak{R}$ then there exists $z \in \mathfrak{R}$ such that $p=a z$ and $q=b z$;

(ii) if $p a=q b$ for some $p, q \in \Re$ then there exists $z \in \mathfrak{R}$ such that $p=z c$ and $q=z d$.

Proof. It follows from the left Ore property that for $a, b \in \mathfrak{R}, b$ regular, there exist $c, d \in \Re, c$ regular, such that $c a=d b$. We can assume that $c$ and $d$ are left coprime. Otherwise, one can simplify on the left by their left greatest common divisor, which is regular since $c$ is.

(i) Let $\mathfrak{I}=\{x \in \mathfrak{R} \mid \exists y \in \mathfrak{R}, d x=c y\}$. $\mathfrak{I}$ is a right ideal in $\mathfrak{R}$, hence it can be written as $h \Re$ for some $h \in \mathfrak{R}$. Obviously $b \in \mathfrak{I}$, thus there exists $g \in \mathfrak{R}$ such that $b=h g$ and both $g, h$ are regular. Element $h$ itself lies in $\mathfrak{I}$, therefore there exists $f \in \mathfrak{R}$ such that $d h=c f$. Multiplying the latter on the right by $g$ we have $c f g=d h g=d b=c a$, which implies that $f g=a$ since $c$ is regular. Recall that $a$ and $b$ are right coprime. Therefore the equalities $a=f g$ and $b=h g$ imply that $g$ is invertible in $\mathfrak{R}$.

Now let us assume that $c p=d q$ for some elements $p, q \in \mathfrak{R}$. By definition of $\mathfrak{I}$ there exists $w \in \mathfrak{R}$ such that $q=h w$. We can rewrite $q$ as $q=h g g^{-1} w=b z$ where $z=g^{-1} w \in \Re$. Finally we note that $c p=d q=d b z=c a z$ which implies $p=a z$ since $c$ is regular.

Taking the left ideal $\mathfrak{J}=\{x \in \mathfrak{R} \mid \exists y \in \mathfrak{R}, x b=y a\}$ we prove part (ii) of the Lemma in a similar way. 


\section{Appendix B. Lemmas Used for the Proof of Proposition 13}

We denote by $\pi$ the projection from the space of Laurent difference polynomials A to the space of difference polynomials K defined by letting $\pi(b)$ being the nonsingular part of $b$ for all difference Laurent monomial $b \in$ A. For example,

$$
\pi\left(u+\frac{u u_{1}}{u_{2}}\right)=u .
$$

If $L=\sum_{n \leq N} l_{n} S^{n}$ is a Laurent series with coefficients being Laurent difference polynomials, we denote by $\pi(L)$ the series $\sum_{n \leq N} \pi\left(l_{n}\right) S^{n}$.

Lemma 6. Let $a, b, c, d \in \mathrm{K}$ and $n \in \mathbb{Z}$. Then $\pi\left[\left(a+b S^{-1}\right) \Delta^{-1}\left(c+d S^{-1}\right)\right]$ is a difference operator.

Proof. We have

$$
\begin{aligned}
\left(a+b S^{-1}\right) \Delta^{-1}\left(c+d S^{-1}\right)= & \sum_{n \geq 1}\left(a c_{-2 n-1} \beta^{n}+b d_{-2 n}\left(\beta^{n-1}\right)_{-1}\right) S^{-2 n-1} \\
& +\sum_{n \geq 0}\left(a d_{-2 n-1} \beta^{n}+b c_{-2 n-2}\left(\beta^{n}\right)_{-1}\right) S^{-2 n-2} \\
& +a c_{-1} \beta^{0} S^{-1} .
\end{aligned}
$$

It is clear that for $n$ large enough $\pi\left(a c_{-2 n-1} \beta^{n}\right)=0$ and similarly $\pi\left(b d_{-2 n}\left(\beta^{n-1}\right)_{-1}\right)=$ 0 .

$$
\pi\left(a c_{-2 n-1} \beta^{n}+b d_{-2 n}\left(\beta^{n-1}\right)_{-1}\right)=0 \text { for } n \gg 0 .
$$

Similarly,

$$
\pi\left(a d_{-2 n-1} \beta^{n}+b c_{-2 n-2}\left(\beta^{n}\right)_{-1}\right)=0 \text { for } n \gg 0 .
$$

Lemma 7. Let $a, b, c, d, \in \mathrm{K}$ and $e \in \mathrm{A}$. Then $\pi\left[\left(a+b S^{-1}\right) \Delta^{-1} e S^{-1} \Delta^{-1}\left(c+d S^{-1}\right)\right]$ is a difference operator.

Proof. Let us expand $L=\left(a+b S^{-1}\right) \Delta^{-1} e S^{-1} \Delta^{-1}\left(c+d S^{-1}\right)$ as a Laurent series in $S^{-1}$ :

$$
\begin{aligned}
L= & \left(a+b S^{-1}\right)\left(\sum_{n \geq 0} \beta^{n} e_{-2 n-1} S^{-2 n-1}\right)\left(\sum_{k \geq 0}\left(\beta^{k}\right)_{-1} S^{-2 k-1}\right)\left(c_{-1} S^{-1}+d_{-1} S^{-2}\right) \\
= & \left(a+b S^{-1}\right)\left(\sum_{m \geq 0} \beta^{m+1}\left(\sum_{n=0}^{m}\left(\frac{e}{u}\right)_{-2 n-1}\right) S^{-2 m-2}\right)\left(c_{-1} S^{-1}+d_{-1} S^{-2}\right) \\
= & \sum_{m \geq 0} a c_{-2 m-3} \beta^{m+1}\left(\sum_{n=0}^{m}\left(\frac{e}{u}\right)_{-2 n-1}\right) S^{-2 m-3} \\
& +\sum_{m \geq 0} b d_{-2 m-4}\left(\beta^{m+1}\right)_{-1}\left(\sum_{n=0}^{m}\left(\frac{e}{u}\right)_{-2 n-2}\right) S^{-2 m-5}
\end{aligned}
$$




$$
\begin{aligned}
& +\sum_{m \geq 0} b c_{-2 m-4}\left(\beta^{m+1}\right)_{-1}\left(\sum_{n=0}^{m}\left(\frac{e}{u}\right)_{-2 n-2}\right) S^{-2 m-4} \\
& +\sum_{m \geq 0} a d_{-2 m-3} \beta^{m+1}\left(\sum_{n=0}^{m}\left(\frac{e}{u}\right)_{-2 n-1}\right) S^{-2 m-4} .
\end{aligned}
$$

After applying $\pi$ to the coefficients of this Laurent series expansion of $L$, we get a difference operator. Let us show it for the first summand in the last line of (65), namely that

$$
\sum_{m \geq 0} \pi\left(a c_{-2 m-3} \beta^{m+1}\left(\sum_{n=0}^{m}\left(\frac{e}{u}\right)_{-2 n-1}\right)\right) S^{-2 m-3}
$$

is a difference operator (the same argument applies to the remaining three summands). This follows from the claim that for large enough $m$, and for all $0 \leq n \leq m$,

$$
\pi\left(a c_{-2 m-3} \beta^{m+1}\left(\frac{e}{u}\right)_{-2 n-1}\right)=0 .
$$

Indeed, if $e$ can be written as a sum of Laurent monomials for which the degree of the numerators, as polynomials in the $u_{i}$ 's are bounded by $m_{e}$, and if $m_{a}$ and $m_{c}$ denote the degrees of $a$ and $c$ as polynomials in the $u_{i}$ 's, then (66) holds for $m>m_{a}+m_{c}+m_{e}$.

Lemma 8. Let $f$ be a difference polynomial such that $R$ is recursion for the equation $u_{t}=f$. Then there exists a difference polynomial $k$ such that $f=u\left(k_{2}-k\right)$.

Proof. Operator $R$ given by (53) is recursion for $u_{t}=f$ which implies that $\ln (u)$ is a conserved density of $f$, or in other words that there is a difference polynomial $g$ such that $f=u\left(g_{1}-g\right)$.

To conclude we need to prove that $g_{1}-g=k_{2}-k$ for some difference polynomial $k$, which is equivalent to say that $g=k_{1}+k+\rho$ for some constant $\rho$. We claim that this is the same as saying that

$$
\sum_{n}(-1)^{n} S^{-n}\left(\frac{\partial g}{\partial u_{n}}\right)=0 .
$$

Indeed, it is clear that $\sum_{n}(-1)^{n} S^{-n} \frac{\partial}{\partial u_{n}}(S+1)=0$ by (3) and that any constant satisfies (67). Conversely, if a difference polynomial $g$ of order $(M, N)$ satisfies (67), then there exists a difference polynomial $k$ and a constant $\rho$ such that $g=k_{1}+k+\rho$. To check this, we proceed by induction on the total order of $g$. If it is zero, meaning that $g$ is a function of $u_{N}$ for a single $N$, then $g$ must be a constant. If not, say if $g$ has order $(M, N)$ with $M<N$, then $\frac{\partial g}{\partial u_{N}}$ does not depend on $u_{M}$. Consequently, we can write $g$ as a sum $h+k$ where $k$ has order $\left(M^{\prime}, N\right)$ with $M<M^{\prime}$ and $h$ has order $\left(M, N^{\prime}\right)$ with $N^{\prime}<N$. Since $g$ and $k+k_{-1}$ both satisfy (67), it follows that $h-k_{-1}$ must satisfy (67) as well, i.e. we reduced the problem to a difference polynomial of lesser total order.

The difference polynomial (67) is the remainder of the division of $g_{*}$ by $(S+1)$ on the left. Let us call it $r$ :

$$
g_{*}=(S+1) X+r, \quad r=\sum_{n}(-1)^{n} S^{-n}\left(\frac{\partial g}{\partial u_{n}}\right),
$$


where $X$ is some difference operator. We want to prove that $r=0$. It is equivalent to prove that the remainder $r^{\prime}$ of the division of $g_{*} u\left(S^{2}-1\right)$ by $S+1$ on the left is 0 . Indeed $r^{\prime}=u r-(u r)_{-2}$ and $r$ is a difference polynomial, therefore $r=0 \Longleftrightarrow r^{\prime}=0$.

We are going to deduce that $r^{\prime}=0$ from the fact that $R$ is recursion for $f=u\left(g_{1}-g\right)$. Note that $f_{*}=u(S-1) g_{*}+g_{1}-g$. Recall Eq. (54) where $R$ was expressed as $\left(Q \Delta^{-1} C+P\right)\left(S^{2}-1\right)^{-1} \frac{1}{u}$. By Definition (36) of a recursion operator we have

$$
\begin{aligned}
& \left(Q_{*}[f]-f_{*} Q\right) \Delta^{-1} C-Q \Delta^{-1} \Delta_{*}[f] \Delta^{-1} C \\
& \quad+Q \Delta^{-1} C_{*}[f]+P(S+1)^{-1} g_{*} u\left(S^{2}-1\right) \\
& \quad-f_{*} P+P_{*}[f]+Q \Delta^{-1} C(S+1)^{-1} g_{*} u\left(S^{2}-1\right)=0 .
\end{aligned}
$$

The idea is to expand (69) as a Laurent series in $S^{-1}$ and to project the coefficients in front of $S^{-N}$ for large $N$ on the space of difference polynomials. Let us start by rearranging (69) using two Euclidean divisions

$$
C=w_{2}+w_{-1}+Z(S+1), \quad g_{*} u\left(S^{2}-1\right)=r^{\prime}+(S+1) Y,
$$

where $Y$ and $Z$ are two difference operators. Combining (69) with (70), we get:

$$
\begin{aligned}
Q \Delta^{-1}\left(w_{2}+w_{-1}\right) S^{2}(S+1)^{-1} r^{\prime}+p(S+1)^{-1} r^{\prime} \\
=Q \Delta^{-1} \Delta_{*}[f] \Delta^{-1} C-\left(Q_{*}[f]-f_{*} Q\right) \Delta^{-1} C \\
\quad-Q \Delta^{-1}\left(C_{*}[f]+C Y+Z r^{\prime}\right)-P r^{\prime}+f_{*} P-P_{*}[f] .
\end{aligned}
$$

By Lemmas 6 and 7, if $M$ is the RHS of (71), $\pi(M)$ is a difference operator. Therefore,

$$
\pi\left[Q \Delta^{-1}\left(w_{2}+w_{-1}\right) S^{2}(S+1)^{-1} r^{\prime}+p(S+1)^{-1} r^{\prime}\right]
$$

must be a difference operator as well. Let us write $Q=a+b S^{-1}$ where $a=u\left(u u_{1}-1\right)$ and $b=u\left(1-u u_{-1}\right)$ and let $c=w_{2}+w_{-1}$. Looking only at even powers of $S^{-1}$ in the Laurent series expansion of (72) we obtain

$$
\begin{aligned}
& \pi\left[\left(a\left(\beta^{0} c_{-1}+\cdots+\beta^{N} c_{-2 N-1}\right)-b\left(\beta^{0} c_{-1}+\cdots\right.\right.\right. \\
& \left.\left.\left.\quad+\beta^{N-1} c_{-2 N+1}\right)_{-1}-p\right) r_{-2 N}^{\prime}\right]=0 \text { for all } N \gg 0,
\end{aligned}
$$

where the Laurent difference polynomials $\beta^{n}=\frac{u_{-1} \ldots u_{-2 n-1}}{u_{\ldots} u_{-2 n}}, n \geq 1, \beta^{0}=\frac{1}{u}$ satisfy

$$
\Delta^{-1}=\sum_{n \geq 0} \beta^{n} S^{-2 n-1} .
$$

It is clear that for all $k>1$ and for all $N \geq \operatorname{Ord} r^{\prime}+2$, we have

$$
\pi\left(a c_{2 k-1} \beta^{k} r_{-2 N}^{\prime}\right)=\pi\left(b\left(c_{2 k-1} \beta^{k}\right)_{-1} r^{\prime}{ }_{-2 N}\right)=0 .
$$

In other words, there exists $K \geq 0$ such that

$$
\begin{aligned}
& \pi\left[\left(a\left(\beta^{0} c_{-1}+\cdots+\beta^{K} c_{-2 K-1}\right)-b\left(\beta^{0} c_{-1}+\cdots\right.\right.\right. \\
& \left.\left.\left.\quad+\beta^{K-1} c_{-2 K+1}\right)_{-1}-p\right) r_{-2 N}^{\prime}\right]=0 \text { for all } N \gg 0 .
\end{aligned}
$$

If $r^{\prime} \neq 0, r^{\prime}$ is either a constant or the order of $r^{\prime}-2 N$ must go to $(-\infty,-\infty)$ as $N$ grows. In both cases we must have:

$$
\pi\left[a\left(\beta^{0} c_{-1}+\cdots+\beta^{K} c_{-2 K-1}\right)-b\left(\beta^{0} c_{-1}+\cdots+\beta^{K-1} c_{-2 K+1}\right)_{-1}-p\right]=0 .
$$


This quantity can be computed directly, and we obtain

$$
\begin{aligned}
& p=-2+u\left(u_{2}+3 u_{1}+2 u+u_{-1}+u_{-2}\right)+2 u_{-1} u_{-3} \\
& -u\left(2 u_{1} u_{-1} u_{-3}+2 u_{1} u u_{-1}+u u_{1} u_{2}+u_{-2} u_{-1} u+2 u_{-4} u_{-2} u\right)
\end{aligned},
$$

which is a contradiction to $p$ given in (57). Thus we have $r^{\prime}=0$ and hence $g=k_{1}+k+\rho$. By now we have proved the statement.

Lemma 9. Let $g \in \mathrm{K}$ be such that $R$ is recursion for $f=u\left(g_{2}-g\right)$. Then

$$
Q \Delta^{-1}\left(C(g)_{*} u\left(S^{2}-1\right)-C(g)\left(S^{2}-S\right)\right)+\left(Q_{*}[f]-f_{*} Q-Q\left(g_{1}-g_{2}\right)\right) \Delta^{-1} C
$$

is a difference operator.

Proof. We have $\Delta_{*}^{-1}[f]=\left(g_{1}-g_{2}\right) \Delta^{-1}+\Delta^{-1}\left(g_{1}-g_{2}\right)$ and $f_{*}=u\left(S^{2}-1\right) g_{*}+g_{2}-g$. From (69) we deduce that

$$
Q \Delta^{-1}\left(C g_{*} u\left(S^{2}-1\right)-\left(g_{2}-g_{1}\right) C+C_{*}[f]\right)+\left(Q_{*}[f]-f_{*} Q-Q\left(g_{2}-g_{1}\right)\right) \Delta^{-1} C
$$

is a difference operator. It remains to rewrite the first nonlocal term. We have modulo left multiplication by $\Delta$ and we have

$$
\begin{aligned}
C g_{*} u\left(S^{2}-1\right) & =C(g)_{*} u\left(S^{2}-1\right)-\left(g_{2}\left(w_{2}\right)_{*}-g_{1}\left(w_{-1}\right)_{*}\right) u\left(S^{2}-1\right) \\
& =C(g)_{*} u\left(S^{2}-1\right)+u_{1} u_{3} g_{2}\left(S^{5}-S\right)-u u_{-2} g_{1}\left(S^{2}-S^{-2}\right) \\
& \equiv C(g)_{*} u\left(S^{2}-1\right)+\left(u u_{-2} g_{-2}-u_{1} u_{3} g_{2}\right) S-u_{1} u_{3}\left(g_{5}-g_{1}\right) S^{2}
\end{aligned}
$$

and

$$
C_{*}[f]=u u_{-2}\left(g_{2}-g_{-2}\right) S-u_{1} u_{3}\left(g_{5}-g_{1}\right) S^{2} .
$$

Therefore

$$
C g_{*} u\left(S^{2}-1\right)-\left(g_{2}-g_{1}\right) C+C_{*}[f] \equiv C(g)_{*} u\left(S^{2}-1\right)-C(g)\left(S^{2}-S\right) .
$$

We conclude combining (78) to (79).

Lemma 10. Let $a, b, c, d, e, f, g, h$ be difference Laurent polynomials such that $a, b, g$, $h \neq 0$ and

$$
\left(a+b S^{-1}\right) \Delta^{-1}\left(c+d S^{-1}\right)+\left(e+f S^{-1}\right) \Delta^{-1}\left(g+h S^{-1}\right)
$$

is a difference operator. Then there exists a constant $\lambda \in \mathrm{k}$ such that

$$
\begin{aligned}
& e+f S^{-1}=\lambda\left(a+b S^{-1}\right) \\
& c+d S^{-1}=-\lambda\left(g+h S^{-1}\right) .
\end{aligned}
$$

Proof. Recall the definition of the Laurent monomials $\beta^{n}$ for $n \geq 0$

$$
\Delta^{-1}=\sum_{n \geq 0} \beta^{n} S^{-2 n-1} .
$$


We have

$$
\begin{aligned}
\left(a+b S^{-1}\right) \Delta^{-1}\left(c+d S^{-1}\right)= & \sum_{n \geq 1}\left(a c_{-2 n-1} \beta^{n}+b d_{-2 n}\left(\beta^{n-1}\right)_{-1}\right) S^{-2 n-1} \\
& +\sum_{n \geq 0}\left(a d_{-2 n-1} \beta^{n}+b c_{-2 n-2}\left(\beta^{n}\right)_{-1}\right) S^{-2 n-2} \\
& +a c_{-1} \beta^{0} S^{-1} .
\end{aligned}
$$

Therefore, we must have for large enough $n$

$$
\begin{aligned}
\beta^{n-1}\left(a d_{-2 n+1}+e h_{-2 n+1}\right)+\left(\beta^{n-1}\right)_{-1}\left(b c_{-2 n}+f g_{-2 n}\right) & =0 \\
\beta^{n}\left(a c_{-2 n-1}+e g_{-2 n-1}\right)+\left(\beta^{n-1}\right)_{-1}\left(b d_{-2 n}+f h_{-2 n}\right) & =0 .
\end{aligned}
$$

Here $\beta^{n}$ has poles at $u, u_{-2}, \ldots, u_{-2 n}\left(\beta^{n}=\frac{u_{-1} \ldots u_{-2 n+1}}{u_{\ldots} u_{-2 n}}\right)$ and $\left(\beta^{n-1}\right)_{-1}$ has poles at $u_{-1}, \ldots, u_{-2 n+1}$. Moreover, the Laurent polynomials inside the parenthesis can only have a bounded number of poles, independently of $n$. Combining these two facts we deduce that for large $n$ the arguments inside the four parenthesis must vanish:

$$
\begin{aligned}
& 0=a d_{-2 n+1}+e h_{-2 n+1} \\
& 0=b c_{-2 n}+f g_{-2 n} \\
& 0=a c_{-2 n-1}+e g_{-2 n-1} \\
& 0=b d_{-2 n}+f h_{-2 n}, \quad n \gg 0 .
\end{aligned}
$$

Since $a, b, g, h \neq 0$, either $e=f=c=d=0$, in which case we can take $\lambda=0$, or $e, f, c, d, \neq 0$. In the latter case we conclude using the fact that, if two Laurent difference polynomials $x$ and $y$ are such that $x_{2 n}=y$ for infinitely many $n \in \mathbb{Z}$, then $x$ and $y$ are both equal to the same constant.

Lemma 11. Let $d$ be a difference polynomial. Then $d$ is in the image of $\Delta$ if and only if

$$
\mathrm{d}_{*} u\left(1+S^{-1}\right)-d=\Delta P,
$$

where $P$ is a difference operator. In this case, we have

$$
d=\Delta\left(-S\left(\sum_{n} \frac{\alpha_{2 n}}{u}\left(\frac{\partial d}{\partial u_{2 n}}\right)_{-2 n}\right)\right) .
$$

Here for all $n \in \mathbb{Z}, \alpha_{2 n}$ (resp. $\alpha_{2 n+1}$ ) is the unique difference Laurent polynomial such that $S^{2 n} u-\alpha_{2 n}$ (resp. $\left.S^{2 n+1} u-\alpha_{2 n+1} S^{-1}\right)$ is divisible on the left by $\Delta$. Moreover,

$$
\sum_{n} \frac{\alpha_{2 n}}{u}\left(\frac{\partial d}{\partial u_{2 n}}\right)_{-2 n}
$$

is a difference polynomial.

Proof. Suppose that $d=u_{1} d_{1}^{\prime}-u d_{-1}^{\prime}$ for a difference Laurent polynomial $d^{\prime}$. Then the Fréchet derivative of $d$ expands as:

$$
\mathrm{d}_{*}=\Delta \mathrm{d}_{*}^{\prime}+d_{1}^{\prime} S-d_{-1}^{\prime} .
$$


Hence (we use to $\equiv$ to denote modulo left multiplication by $\Delta$ ) we get

$$
\mathrm{d}_{*} u\left(1+S^{-1}\right) \equiv\left(S d^{\prime}-d_{-1}^{\prime}\right) u\left(1+S^{-1}\right) \equiv u_{1} d_{1}^{\prime}-u d_{-1}^{\prime} \equiv d .
$$

Conversely assume that

$$
\mathrm{d}_{*} u\left(1+S^{-1}\right) \equiv d .
$$

Recall that the $\alpha_{n}$ 's are defined so that $S^{2 n} u \equiv \alpha_{2 n}$ and $S^{2 n+1} u \equiv \alpha_{2 n+1} S^{-1}$ for all $n \in \mathbb{Z}$. The following identity can be easily checked by induction

$$
\alpha_{2 n+2}=\frac{u}{u_{-1}} \alpha_{2 n+1}=\frac{u^{2}}{u_{-1} u_{-2}}\left(\alpha_{2 n}\right)_{-2}, \quad \forall n \in \mathbb{Z} .
$$

Let us rewrite the LHS of (85):

$$
\begin{aligned}
\sum_{n} & S^{n}\left(\frac{\partial d}{\partial u_{n}}\right)_{-n} u\left(1+S^{-1}\right) \\
= & \sum_{n} S^{n} u\left(\left(\frac{\partial d}{\partial u_{n}}\right)_{-n}+\frac{u_{1}}{u}\left(\frac{\partial d}{\partial u_{n+1}}\right)_{-n}\right) \\
\equiv & \sum_{n} \alpha_{2 n}\left(\left(\frac{\partial d}{\partial u_{2 n}}\right)_{-2 n}+\frac{u_{1}}{u}\left(\frac{\partial d}{\partial u_{2 n+1}}\right)_{-2 n}\right) \\
& +\sum_{n} \alpha_{2 n+1} S^{-1}\left(\left(\frac{\partial d}{\partial u_{2 n+1}}\right)_{-2 n-1}+\frac{u_{1}}{u}\left(\frac{\partial d}{\partial u_{2 n+2}}\right)_{-2 n-1}\right) .
\end{aligned}
$$

Combining (85), (86) and (87) we obtain

$$
\begin{aligned}
& d=\sum_{n} \alpha_{2 n}\left(\frac{\partial d}{\partial u_{2 n}}\right)_{-2 n}+\frac{u_{1}}{u} \sum_{n} \alpha_{2 n}\left(\frac{\partial d}{\partial u_{2 n+1}}\right)_{-2 n}, \\
& 0=\sum_{n} \frac{u}{u_{-2}}\left(\alpha_{2 n}\right)_{-2}\left(\frac{\partial d}{\partial u_{2 n+1}}\right)_{-2 n-2}+\sum_{n} \alpha_{2 n+2}\left(\frac{\partial d}{\partial u_{2 n+2}}\right)_{-2 n-2}
\end{aligned}
$$

from which it follows that

$$
\begin{aligned}
d & =\Delta\left(-\sum_{n} \frac{\left(\alpha_{2 n}\right)_{-1}}{u_{-1}}\left(\frac{\partial d}{\partial u_{2 n+1}}\right)_{-2 n-1}\right) \\
& =\Delta\left(-S\left(\sum_{n} \frac{\alpha_{2 n}}{u}\left(\frac{\partial d}{\partial u_{2 n}}\right)_{-2 n}\right)\right) .
\end{aligned}
$$

We proved that there exists a Laurent difference polynomial $d^{\prime}$ such that $d=u_{1} d_{1}^{\prime}-$ $u d_{-1}^{\prime}$. It implies that $d^{\prime}$ cannot have poles (since its highest pole should be lesser or equal than 0 and its lowest pole should be greater than 0 ), therefore that it is a difference polynomial. 


\section{References}

1. Ablowitz, M.J., Kaup, D.J., Newell, A.C., Segur, H.: Inverse scattering transform-Fourier analysis for nonlinear problems. Stud. Appl. Math. 53(4), 249-315 (1974)

2. Olver, P.J.: Evolution equations possessing infinitely many symmetries. J. Math. Phys. 18(6), 1212-1215 (1977)

3. Fuchssteiner, B.: Application of hereditary symmetries to nonlinear evolution equations. Nonlinear Anal. Theory Methods Appl. 3(11), 849-862 (1979)

4. Magri, F.: A Geometrical Approach to the Nonlinear Solvable Equations. Volume 120 of Lecture Notes in Physics, pp. 233-263. Springer (1980)

5. Sanders, J.A., Wang, J.P.: Integrable systems and their recursion operators. Nonlinear Anal. 47, 5213$5240(2001)$

6. Sergyeyev, A.: Why nonlocal recursion operators produce local symmetries: new results and applications. J. Phys. A: Math. Gen. 38, 3397-3407 (2005)

7. Wang, J.P.: Lenard scheme for two-dimensional periodic Volterra chain. J. Math. Phys. 50, 023506 (2009)

8. Gel'fand, I.M., Dorfman, I.Y.: Hamiltonian operators and algebraic structures related to them. Funct. Anal. Appl. 13(4), 248-262 (1979)

9. Dorfman, I.: Dirac Structures and Integrability of Nonlinear Evolution Equations. Wiley, Chichester (1993)

10. Fokas, A.S., Fuchssteiner, B.: On the structure of symplectic operators and hereditary symmetries. Lett. Nuovo Cimento (2) 28(8), 299-303 (1980)

11. Fuchssteiner, B., Fokas, A.S.: Symplectic structures, their Bäcklund transformations and hereditary symmetries. Phys. D 4(1), 47-66 (1981)

12. Kac, V.G., Barakat, A., De Sole, A.: Poisson vertex algebras in the theory of Hamiltonian equations. Jpn. J. Math. 4, 141-252 (2009)

13. Kac, V.G., De Sole, A.: Non-local Poisson structures and applications to the theory of integrable systems. Jpn. J. Math. 8, 233-347 (2013)

14. Valeri, D., De Sole, A., Kac, V.G.: A new scheme of integrability for (bi)Hamiltonian PDE. Commun. Math. Phys. 347, 449-488 (2016)

15. Kupershmidt, B.A.: Discrete Lax Equations and Differential-Difference Calculus, Volume 123 of Astérisque. Société mathématique de France, Paris (1985)

16. Magri, F.: A simple model of integrable Hamiltonian equation. J. Math. Phys. 19(5), 1156-1162 (1978)

17. Carpentier, S.: A sufficient condition for a rational differential operator to generate an integrable system. Jpn. J. Math. 12, 33-89 (2017)

18. Adler, V.E., Postnikov, V.V.: Differentialdifference equations associated with the fractional Lax operators. J. Phys. A Math. Theor. 44(41), 415203 (2011)

19. Ablowitz, M.J., Ladik, J.F.: Nonlinear differential-difference equations and Fourier analysis. J. Math. Phys. 17(6), 1011-1018 (1976)

20. Khanizadeh, F., Mikhailov, A.V., Wang, J.P.: Darboux transformations and recursion operators for differential-difference equations. Theor. Math. Phys. 177(3), 1606-1654 (2013)

21. Ya, A., Maltsev and S.P. Novikov: On the local systems Hamiltonian in the weakly nonlocal Poisson brackets. Physica D: Nonlinear Phenomena 156(1-2), 53-80 (2001)

22. Artin, E.: Geometric Algebra. Interscience Publ, New York (1957)

23. Draxl, P.K.: Skew Fields. London Mathematical Society Lecture Note Series. Cambridge University Press (1983)

24. McConnell, J.C., Robson, J.C., Small, L.W.: Noncommutative Noetherian Rings. Graduate Studies in Mathematics. American Mathematical Society, Providence (2001)

25. Carpentier, S., De Sole, A., Kac, V.G.: Some remarks on non-commutative principal ideal rings. C. R. Math. 351(1), 5-8 (2013)

26. Taelman, L.: Dieudonné determinants for skew polynomial rings. J. Algebra Appl. 05(01), 89-93 (2006)

27. Dieudonné, M.J.: Les déterminants sur un corps non commutatif. Bull. Soc. Math. Fr. 71, $27-45$ (1943)

28. Zhiber, A.V., Sokolov, V.V.: Exactly integrable hyperbolic equations of Liouville type. Uspekhi Mat. Nauk 56(1(337)), 63-106 (2001)

29. Sanders, J.A., Wang, J.P.: On a family of operators and their Lie algebras. J. Lie Theory 12(2), 503-514 (2002)

30. Sanders, J.A., Wang, J.P.: On the integrability of homogeneous scalar evolution equations. J. Differ. Equ. 147(2), 410-434 (1998)

31. Mikhailov, A.V., Novikov, V.S., Wang, J.P.: Symbolic representation and classification of integrable systems. In: MacCallum, M.A.H., Mikhailov, A.V. (eds.) Algebraic Theory of Differential Equations, pp. 156-216. Cambridge University Press, Cambridge (2009)

32. Kiselev, A.V., van de Leur, J.W.: Symmetry algebras of Lagrangian Liouville-type systems. Theor. Math. Phys. 162(2), 149-162 (2010) 
33. Kiselev, A.V., van de Leur, J.W.: Pre-Hamiltonian structures for integrable nonlinear systems. arXiv:math-ph/0703082v1

34. Mikhailov, A.V., Carpentier, S., Wang, J.P.: PreHamiltonian and Hamiltonian Operators for Integrable Differential-Difference Equations (2018). arXiv:math-ph/1808.02957

35. Mansfield, E., Beffa, G.M., Wang, J.P.: Discrete moving frames and discrete integrable systems. Found. Comput. Math. 13(4), 545-582 (2013)

36. Mikhailov, A.V., Wang, J.P., Xenitidis, P.: Cosymmetries and Nijenhuis recursion operators for difference equations. Nonlinearity 24(7), 2079-2097 (2011). arXiv:1009.2403

37. Wang, J.P.: Recursion operator of the Narita-Itoh-Bogoyavlensky lattice. Stud. Appl. Math. 129(3), 309327 (2012)

38. Mikhailov, A.V., Sokolov, V.V.: Integrable odes on associative algebras. Commun. Math. Phys. 211(1), 231-251 (2000)

39. Fuchssteiner, B., Oevel, W., Wiwianka, W.: Computer-algebra methods for investigation of hereditary operators of higher order soliton equations. Comput. Phys. Commun. 44(1-2), 47-55 (1987)

40. Hereman, W., Sanders, J.A., Sayers, J., Wang, J.P.: Symbolic computation of polynomial conserved densities, generalized symmetries, and recursion operators for nonlinear differential-difference equations. In: Group Theory and Numerical Analysis Book series title: CRM proceedings and Lecture Notes, vol. 39, pp. 133-148. Amer. Math. Soc., Providence (2005)

41. Gürses, M., Karasu, A., Sokolov, V.V.: On construction of recursion operators from Lax representation. J. Math. Phys. 40(12), 6473-6490 (1999)

42. Zhang, D., Chen, D.: Hamiltonian structure of discrete soliton systems. J. Phys. A Math. Gen. 35(33), 7225-7241 (2002)

43. Toda, M.: Wave propagation in anharmonic lattices. J. Phys. Soc. Jpn. 23(3), 501-506 (1967)

44. Flaschka, H.: The Toda lattice. II. Existence of integrals. Phys. Rev. B 9, 1924-1925 (1974)

45. Manakov, S.V.: Complete integrability and stochastization in discrete dynamical systems. Sov. Phys. JETP 40, 269-274 (1975)

46. Ruijsenaars, S.N.M.: Relativistic Toda systems. Commun. Math. Phys. 133(2), 217-247 (1990)

47. Oevel, W., Fuchssteiner, B., Zhang, H., Ragnisco, O.: Mastersymmetries, angle variables, and recursion operator of the relativistic Toda lattice. J. Math. Phys. 30(11), 2664-2670 (1989)

48. Zhang, H., Gui-Zhang, T., Oevel, W., Fuchssteiner, B.: Symmetries, conserved quantities, and hierarchies for some lattice systems with soliton structure. J. Math. Phys. 32(7), 1908-1918 (1991)

49. Tsuchida, T.: Integrable discretizations of derivative nonlinear Schrödinger equations. J. Phys. A: Math. Gen. 35(36), 7827-7847 (2002)

50. Marí Beffa, G., Sanders, J.A., Wang, J.P.: Integrable systems in three-dimensional Riemannian geometry. J. Nonlinear Sci. 12(2), 143-167 (2002)

Communicated by Y. Kawahigashi 